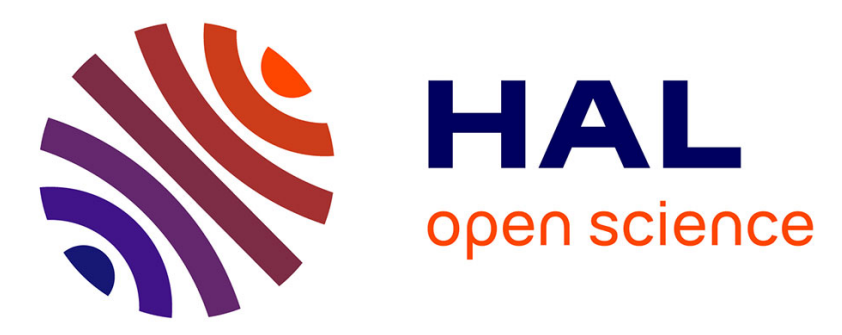

\title{
Vaisselle de terre et pouvoirs politiques à Chypre aux époques comnène, franque et vénitienne
}

Véronique François

\section{To cite this version:}

Véronique François. Vaisselle de terre et pouvoirs politiques à Chypre aux époques comnène, franque et vénitienne. Cahiers du Centre d'Etudes Chypriotes, 2013, 43, pp.423-454. halshs-01105220

\section{HAL Id: halshs-01105220 \\ https://shs.hal.science/halshs-01105220}

Submitted on 30 May 2017

HAL is a multi-disciplinary open access archive for the deposit and dissemination of scientific research documents, whether they are published or not. The documents may come from teaching and research institutions in France or abroad, or from public or private research centers.
L'archive ouverte pluridisciplinaire $\mathbf{H A L}$, est destinée au dépôt et à la diffusion de documents scientifiques de niveau recherche, publiés ou non, émanant des établissements d'enseignement et de recherche français ou étrangers, des laboratoires publics ou privés. 


\title{
Vaisselle de terre et pouvoirs politiques à Chypre aux époques comnène, franque et vénitienne Véronique François
}

\begin{abstract}
The comparative study of the written sources, of the literary, architectural, artistic and archaeological data has highlighted a Frankish identity in Cyprus between the late twelfth and early sixteenth century. The question that arises here is whether the Frankish identity appeared also in the pottery used in the island at the time of the kingdom of Lusignan. To measure the impact that the Latin presence has had on the production and the supply of pottery in Frankish Cyprus, it is necessary to examine the situation before the arrival of the Latins when the island was a Byzantine province in which the potters did not manufactured glazed ceramic. Considering this situation, the creation ex-nihilo of workshops in the middle of the thirteenth century asks the question of the origin of artisans. Until now the Medieval Cypriot pottery was considered as a production under Byzantine influence. However the fine glazed wares were probably more connected with the Christian Levant than with Byzantium. We shall also consider the evolution of the pottery trade after the conquest of the island by the Venetians in the late fifteenth century. Thus, in this well-defined framework, we will try to give a definition of the " Frankish Pottery" in Cyprus, terms that refer to different realities during the thirteenth, fourteenth and fifteenth centuries.
\end{abstract}

\section{Citer ce document / Cite this document :}

François Véronique. Vaisselle de terre et pouvoirs politiques à Chypre aux époques comnène, franque et vénitienne. In: Cahiers du Centre d'Etudes Chypriotes. Volume 43, 2013. pp. 423-454;

http://www.persee.fr/doc/cchyp_0761-8271_2013_num_43_1_1078 


\title{
VAISSELLE DE TERRE ET POUVOIRS POLITIQUES À CHYPRE AUX ÉPOQUES COMNÈNE, FRANQUE ET VÉNITIENNE
}

\author{
Véronique FRANÇOIS
}

\begin{abstract}
The comparative study of the written sources, of the literary, architectural, artistic and archaeological data has highlighted a Frankish identity in Cyprus between the late twelfth and early sixteenth century. The question that arises here is whether the Frankish identity appeared also in the pottery used in the island at the time of the kingdom of Lusignan. To measure the impact that the Latin presence has had on the production and the supply of pottery in Frankish Cyprus, it is necessary to examine the situation before the arrival of the Latins when the island was a Byzantine province in which the potters did not manufactured glazed ceramic. Considering this situation, the creation ex-nihilo of workshops in the middle of the thirteenth century asks the question of the origin of artisans. Until now the Medieval Cypriot pottery was considered as a production under Byzantine influence. However the fine glazed wares were probably more connected with the Christian Levant than with Byzantium. We shall also consider the evolution of the pottery trade after the conquest of the island by the Venetians in the late fifteenth century. Thus, in this welldefined framework, we will try to give a definition of the "Frankish Pottery" in Cyprus, terms that refer to different realities during the thirteenth, fourteenth and fifteenth centuries.
\end{abstract}

L'examen et la confrontation des sources historiques, littéraires, architecturales, artistiques et archéologiques permettent la mise en évidence de l'identité franque à Chypre entre la fin du $\mathrm{XII}^{\mathrm{e}}$ et le début $\mathrm{du} \mathrm{XVI}^{\mathrm{e}}$ siècle. La question qui se pose ici est de savoir si cette identité franque s'est manifestée dans la vaisselle de terre employée dans l'île à l'époque du royaume des Lusignan. Afin de mesurer l'impact que la présence latine a pu avoir sur la production et sur l'approvisionnement en poteries de Chypre franque, il convient d'examiner la situation avant l'arrivée des Latins, alors que l'île était une province byzantine dans laquelle aucun potier ne fabriquait encore de céramique glaçurée. Compte tenu de cette situation, la création ex nihilo d'ateliers au milieu du XIII ${ }^{\mathrm{e}}$ siècle pose la question de l'origine des artisans. Jusqu'alors considérée comme une production d'influence byzantine, la vaisselle fine d'époque franque tient sans doute plus du Levant chrétien que de Byzance. Afin de montrer en quoi cette production était particulière sous les Lusignan, nous verrons aussi quelle a été l'évolution du marché de la vaisselle après la 
conquête de l'île par les Vénitiens à la fin du $\mathrm{Xv}^{\mathrm{e}}$ siècle. Ainsi, dans ce cadre bien défini, nous nous interrogerons sur la définition de la « vaisselle franque » à Chypre, des termes qui renvoient à des réalités bien différentes au XIII ${ }^{\mathrm{e}}$, au XIV et au XV siècles.

\section{I. Évolution du « vaisselier » à Chypre, du XII ${ }^{\mathrm{e}}$ au XVI ${ }^{\mathrm{e}}$ siècle}

\section{1. À la veille de la conquête franque : prépondérance des importations de Byzance et de l'Orient islamique}

Tête de pont de l'Empire byzantin au Levant, Chypre, sous les Comnène, a joui d'une période de prospérité économique et démographique sans précédent ${ }^{1}$. L'île était bien peuplée : la population, estimée à 100.000 habitants, était, pour l'essentiel, composée de Grecs orthodoxes, mais aussi de juifs, de coptes, d'Arméniens, d'une communauté arabe, de paysans et de marchands syriens melkites, et de commerçants italiens. Chypre était bien pourvue en richesses naturelles ; l'élevage, l'agriculture et la viticulture étaient florissants et permettaient d'approvisionner les États croisés. En effet, la première Croisade (1096-1099) et l'installation des Latins en Syrie et en Palestine ont contribué au développement et à la richesse de l'île. C'était une étape pour les pèlerins qui se rendaient en Terre Sainte par voie maritime, elle se trouvait sur la route des croisés et c'était une halte pour les ambassades byzantines, envoyées dans le comté de Tripoli, la principauté d'Antioche ou le royaume de Jérusalem. Des liaisons maritimes étaient établies entre Chypre et les villes côtières d'Antioche, de Tripoli, de Jaffa et de Saint-Jean-d'Acre ${ }^{2}$. L'île accueillait des marchands étrangers, vénitiens surtout - les compagnies vénitiennes installées à Paphos et Limassol commerçaient avec Acre, Damiette et Alexandrie ${ }^{3}$. Toute cette activité a contribué à l'essor des ports. Celui de Paphos, point d'embarquement pour la Terre Sainte, était le plus grand de l'île, mais il fallait également compter avec le port de Limassol qui desservait la Syrie, et les ports de Famagouste et de Kyrenia. Les marchés, selon le géographe arabe Al-Idrisi, étaient bien pourvus en « toute sorte de produits, des biens manufacturés et des marchandises ${ }^{4}$. Les acheteurs y trouvaient des objets issus de l'artisanat local, tels que des pièces d'orfèvrerie, des croix-pendentifs ou de petites icônes en stéatite ${ }^{5}$, des tissus et notamment de la soie, des broderies et des poteries. Cependant l'archéologie nous révèle que la gamme des terres cuites offertes à la vente au XII ${ }^{\mathrm{e}}$ siècle se limitait à une série de céramiques culinaires et de stockage assez grossières, produites

1. Sur l'histoire de Chypre à l'époque de la seconde domination byzantine, de 965 à 1191 , et sur les sources disponibles pour cette période voir Malamut 2012, p. 96-102 ; Cheynet 1993, p. 67-77.

2. Malamut 1988, vol. II, p. 557.

3. Papacostas 2006 ; Malamut 1988, vol. I, p. 253, vol. II, p. 557-558.

4. Géographie d'Edrisi, p. 130.

5. Les fouilles du Palaion Demarcheion à Nicosie ont mis au jour les indices d'un atelier de taille de stéatite en activité au XII siècle : Violaris 2004, p. 69-80 ; Durand, Giovannoni 2012, p. 155 et 168-173. 
localement. La clientèle qui souhaitait équiper sa maison en vaisselle de table n'avait pas d'autre choix que d'acheter des coupes et des plats importés du Proche-Orient et des ateliers des Balkans et d'Asie Mineure.

Les fouilles récentes d'un quartier d'habitation médiéval situé au cœur de la vieille ville de Nicosie sur le site du Palaion Demarcheion ont livré un bel échantillonnage des ustensiles de terre employés dans l'île sous les Comnène ${ }^{6}$. Cet ensemble clos, qui constitue le comblement d'une fosse, comprend des céramiques communes d'origines locales. La vaisselle culinaire consiste en grosses marmites tournées, à panse globulaire, à deux anses, ornées parfois de cordons appliqués digités, réalisées dans une pâte granuleuse et ferrugineuse, dure, rouge foncé, qui est aussi employée pour des petits pots de cuisson tournés et des jattes allant au feu, modelées, à lèvre rentrante ${ }^{7}$. Des jarres tournées et des amphorettes servaient pour le stockage. Des bassins à tout faire et des godets de noria complètent l'inventaire des poteries communes ${ }^{8}$. Ces objets aux fonctions variées étaient fabriqués dans l'île, sans doute dans les ateliers de Kornos ou de Phoini.

Kornos, connu durant la période latine sous le nom de Cornos, est un petit village, situé sur les contreforts orientaux du massif du Troodos ${ }^{9}$ et, jusqu'à ces dernières années, les femmes de ce bourg, qui ont derrière elles une longue tradition du travail de la terre, ont conservé une technique de fabrication entièrement montée à la main ${ }^{10}$. Le village de Phoini est implanté à $1000 \mathrm{~m}$ d'altitude sur le versant sud du Troodos ; il est réputé pour sa fabrication de pitharia, des grandes jarres pour le vin. La terre grossière, employée jusqu'il y a peu dans ces ateliers, semble être la même que celle des objets archéologiques. Capable de résister aux chocs thermiques, elle est utilisée pour les pots culinaires mais sa texture poreuse favorise l'évaporation et la conservation de l'eau fraîche ou des grains, elle convient donc aussi à l'élaboration de grosses jarres rondes de stockage inamovibles et de petits vases à liquide. La permanence entre les formes des céramiques communes à pâte rouge retrouvées dans les fouilles et les prospections, dans des contextes datés entre le $\mathrm{XII}^{\mathrm{e}}$ et le $\mathrm{XVI}^{\mathrm{e}}$ siècle, et celles des poteries modernes est confondante, c'est pourquoi il est tentant d'attribuer la production médiévale à ces villages ${ }^{11}$.

Une autre partie de l'assemblage de Nicosie - des jattes allant au feu et une marmite à panse profonde fabriquées dans une pâte rouge brunâtre et couvertes, à l'intérieur, d'une glaçure plombifère brun foncé - est sans doute originaire des ateliers de Beyrouth ${ }^{12}$,

6. Violaris 2004, p. 69-80 ; Wartburg, Violaris 2009, p. 249-254.

7. Wartburg, Violaris 2009, p. 251, fig. 2.

8. Wartburg, Violaris 2009, p. 252, fig. 3.

9. Grivaud 1998, p. 461 ; Ionas 2000.

10. Les céramiques sont modelées. La tournette est employée pour les différentes phases de la finition.

11. Le stock très imposant vendu dans le village de Mosphiloti conserve le souvenir des productions du siècle dernier et constitue un véritable conservatoire du patrimoine potier de Chypre.

12. François et al. 2003, p. 325-340. 
dont les productions étaient commercialisées dès l'époque fatimide, comme l'atteste la cargaison de l'épave de Serçe Limanı ${ }^{13}$. Pour sa part, la vaisselle de table et de service retrouvée dans cette fosse est exclusivement d'origine étrangère.

Des Fine Sgraffito Wares fabriquées dans des ateliers de l'Empire byzantin, peutêtre dans le Péloponnèse, côtoient des productions réalisées par des potiers ayyoubides d'Égypte ou de Syrie - des coupes à pâte argileuse, engobées, peintes au manganèse sous une glaçure alcaline incolore ou bleue, ornées d'élégants rinceaux végétaux, et des pots et des coupelles à pâte argileuse couverte d'une glaçure alcaline verte ou aubergine, parfois appliquée sur engobe. Ces importations orientales ne sont pas exceptionnelles. Découvertes dans un contexte urbain à Nicosie, d'autres trouvailles témoignent de leur utilisation sur des sites de natures variées. Elles sont apparues en effet dans les fouilles du monastère d'Ayia Moni au pied du Troodos, à Kouklia et à Leptos Walls à proximité du port de Paphos ${ }^{14}$. Ces importations sont représentées par des coupes à pâte siliceuse dure, sur laquelle est appliquée une glaçure alcaline incolore, parfois rehaussée de coulures bleu de cobalt, et des cruches et des coupes, en pâte siliceuse plus tendre, à glaçure alcaline bleu turquoise ou vert pâle ; par des plats à pâte argileuse, tantôt couverts d'une glaçure alcaline aubergine ou bleue, tantôt peints au lustre métallique jaune d'or ou rouge (Fig.1.1), tandis que d'autres récipients sont ornés d'un décor géométrique sommairement incisé ${ }^{15}$. Ces pièces sont identifiées comme des productions islamiques du $\mathrm{XII}^{\mathrm{e}}$ siècle, mais il n'est pas exclu, en fait, que certaines d'entre elles datent plutôt du XI ${ }^{\mathrm{e}}$. Elles attestent l'importance de la vaisselle islamique, sans doute dès l'époque fatimide, dans l'instrumentum chypriote.

Ces trouvailles encore peu nombreuses témoignent de l'existence, à l'époque comnène, d'un vaisselier composite qui illustre bien la position stratégique occupée par Chypre entre monde byzantin et Proche-Orient islamique. Aux XI et XII ${ }^{\mathrm{e}}$ siècles, les céramiques communes, destinées à la préparation et à la cuisson des aliments, ainsi qu'au stockage, étaient fabriquées localement. Cependant des ustensiles de mêmes fonctions, réalisés à Beyrouth, complétaient la batterie de cuisine. Assez curieusement, pour cette époque, on ne trouve aucune trace de production locale de vaisselle glaçurée pour le service des mets. Les objets remplissant cette fonction proviennent pour l'essentiel du monde islamique, sans doute de Syrie, mais peut-être aussi d'Égypte et, de façon plus marginale, d'ateliers d'Asie Mineure ou des Balkans. Ainsi, l'approvisionnement en vaisselle à Chypre témoigne, pour les $\mathrm{XI}^{\mathrm{e}}$ et XII ${ }^{\mathrm{e}}$ siècles, des liens que l'île entretenait déjà avec le Proche-Orient. Des contacts culturels étaient établis entre Chypre et la SyriePalestine-des parentés stylistiques existent entre des fresques chypriotes et des miniatures de l'Antiochène ${ }^{16}$; des modèles identiques dans l'écriture (le «style epsilon »), dans

13. Waksman 2011, p. 201-212.

14. Wartburg 2003, p. 153-166 ; Wartburg et al. 2010, p. 390-410.

15. Wartburg 2003, p. 154, 155, fig. 2, 3.2, 3.4 et 3.6, fig. 3, 3.3 et 3.5 .

16. Weyl Carr 2005, p. 286. 
l'ornementation et dans l'iconographie, se retrouvent dans des manuscrits de Palestine et de Chypre ${ }^{17}$. Cependant, dans l'artisanat céramique, on n'observe pas encore d'influence entre ces deux univers. La situation changera au milieu du XIII ${ }^{\mathrm{e}}$ siècle.

\section{Sous les Lusignan : caractère cosmopolite du vaisselier et création ex-nihilo d'ateliers de potier}

Les bouleversements politiques survenus à la fin du XII ${ }^{\mathrm{e}}$ siècle - en 1192, l'île a été vendue par Richard Cour de Lion à Guy de Lusignan, roi de Jérusalem contesté - et l'installation à Chypre d'une population de culture franque, venue pour l'essentiel du Levant, ont eu des conséquences sur le marché de la vaisselle. À partir de la deuxième moitié du XIII ${ }^{\mathrm{e}}$ siècle, des potiers, probablement originaires du Levant, se sont installés dans l'île pour offrir à la clientèle locale de la vaisselle de service glaçurée. Par ailleurs, alors que Chypre était devenue un entrepôt du commerce entre l'Europe, la Méditerranée orientale et le Proche-Orient, l'approvisionnement en poteries étrangères s'est encore diversifié.

De la fin du XII ${ }^{\mathrm{e}}$ siècle jusqu'aux premières années du XIV ${ }^{\mathrm{e}}$, la batterie de cuisine « franque » était, comme sous les Comnène, constituée d'une part de marmites à panse globulaire, de jattes allant au feu, de coupes, de pots et de cruches à bec pincé, modelés dans une argile rouge-brunâtre, fabriqués localement et, d'autre part, de jattes culinaires et de marmites glaçurées des ateliers de Beyrouth (Fig.1 .2). Cette association de céramiques culinaires chypriotes et berythaines est attestée dans plusieurs fouilles : dans deux fosses, datées de la fin $\mathrm{XII}^{\mathrm{e}}$ ou du début du XIII ${ }^{\mathrm{e}}$ siècle, à Kouklia ${ }^{18}$; à Paphos, dans les niveaux de destruction du début du XIII ${ }^{\mathrm{e}}$ siècle du château de Saranda Kolones ${ }^{19}$, dans Icarus Street pour la deuxième moitié du XIII ${ }^{\mathrm{e}}$ siècle ${ }^{20}$, et dans un puits sur la colline Fabrika dans les dernières années du XIII ${ }^{\mathrm{e}}$ ou les premières années du XIV ${ }^{\mathrm{e}}$ siècle ${ }^{21}$; à Kato Paphos, dans une fouille de sauvetage près de l'église Agia Théosképasti ${ }^{22}$. À l'évidence, il existait à Chypre un marché pour la production des potiers levantins. Il est possible, comme S. Gabrielli en fait l'hypothèse, que des relations étroites, parfois familiales, liaient les

17. Yota 2012, p. 138-149.

18. Wartburg 1997a, p. 187, fig. 11, 11-12, et p. 190, fig. 15, 22-25.

19. Megaw 1972, p. 334, fig. D, pot type A, fig. E, pot type B, fig. 23, 24, 27 ; Megaw, Rosser 2001, p. 319-331, p. 325, fig. 6, 10, 11, 13, et p. 327, fig. 7, 26.

20. Gabrielli 2008, p. 423-454. Les analyses chimiques des pâtes d'un matériel identique trouvé à Leptos Walls et à Kouklia confirment une fabrication dans les environs de Beyrouth : Waksman 2002, p. 67-77.

21. Sur ce site, un complexe artisanal, sans doute détruit par un tremblement de terre, regroupait des artisans du verre, du métal et des potiers qui réalisaient des céramiques incisées ou peintes à l'engobe de même type que celles des ateliers de Paphos-Lemba : Gabrielli, McCall, Green 2001, p. 335-353; Cook, Green 2002, p. 413-426.

22. Une monnaie datée du XIII ${ }^{\mathrm{e}}$ siècle fournit un terminus post quem pour ce contexte: Wartburg 2003, p. 160 et 162, fig. 12 . 
populations côtières des deux côtés de la mer ${ }^{23}$. Sur la base du différentiel de coût entre transport maritime et transport terrestre, le commerce d'un port à l'autre était sans doute plus aisé que vers l'intérieur des terres. Ainsi un potier de Beyrouth pouvait avoir plus de facilité pour distribuer sa production à Famagouste que pour l'envoyer à Jérusalem. Les villes côtières, levantines et chypriotes, bénéficiaient sans doute d'un même réseau commercial dont la distribution de la céramique culinaire témoigne.

Les importations de vaisselle fine sont représentées à parts égales entre des céramiques byzantines et une grande variété de productions islamiques. L'exploration archéologique de Paphos - Saranda Kolones ${ }^{24}$, Fabrika, Leptos Walls ${ }^{25}$ et Garrison's Camp $^{26}$-, ainsi que les fouilles de Kouklia ${ }^{27}$ et de Limassol ${ }^{28}$, ont livré des exemplaires de Zeuxippus Ware et d'Aegean Ware, des coupes incisées et glaçurées datées du milieu $\mathrm{XII}^{\mathrm{e}}$-troisième quart du $\mathrm{XIII}^{\mathrm{e}}$ siècle, dont les origines exactes ne sont pas connues (Fig. 1.35). Ces céramiques byzantines étaient très largement commercialisées à travers toute la Méditerranée, y compris au Proche-Orient ${ }^{29}$. Les productions islamiques, pour la plupart d'origines syriennes, sont encore présentes au XIII ${ }^{\mathrm{e}}$ siècle à Paphos sous la forme d'un albarelle, d'une base de jarre à pâte siliceuse et d'une coupe au château de Saranda Kolones, ou de petits fragments de coupe sur la colline Fabrika ${ }^{30}$.

Cependant de nouveaux produits ont fait leur apparition. Ainsi trouve-t-on la trace, à Nicosie ${ }^{31}$, mais aussi à Paphos, à Kouklia et à Limassol ${ }^{32}$, des poteries dites Saint Symeon Wares ou céramiques «d'Al-Mina » (Fig. 1.6.7). Il s'agit de coupes à pâte argileuse, incisées et rehaussées de pigments colorés sous une glaçure plombifère. Fabriquées dans la région d'Antioche et plus largement dans plusieurs ateliers de Cilicie et du nord de la Syrie, ce type de vaisselle était employé à Chypre, dans l'Empire byzantin, ainsi que sur les côtes de Syrie-Palestine ${ }^{33}$. Ces vases d'origine orientale entraient désormais en concurrence avec des produits d'un tout autre aspect, venus cette fois du bassin occidental de la Méditerranée. En effet des faïences - c'est-à-dire des pâtes argileuses glaçurées à

23. Gabrielli 2007, p. 403.

24. Megaw, Rosser 2001, p. 319-331.

25. Wartburg 2003, p. 153-166.

26. Giudice et al. 1994, p. 256-260, pl. LXXI, 1-2 ; Giudice et al. 1996, p. 240, pl. XXXV, 14.

27. Wartburg 1998, p. 133-165.

28. Prokopiou 1997, p. 285-322.

29. François 2012, p. 51 carte 1 et p. 52 carte 3.

30. Megaw 1972, p. 336, fig. 29 ; Megaw, Rosser 2001, p. 328, fig. 8, 41, et p. 329 ; François 2011.

31. Flourentzos 1994, pl. XXVI, 60.

32. Giudice et al. 1996, p. 248, pl. XXXVIII, 3 ; Wartburg 2003, p. 161-162 fig. 18, 22- 24.

33. Des ratés de cuisson ont été découverts à Al-Mina, le port d'Antioche, mais aussi à Misis en Cilicie, dans une forteresse des Templiers à Kinet, dans le golfe d'Alexandrette, et à Épiphania (25 km au nord de Kinet) : Lane 1937 ; Hild, Hellenkemper 1990 ; Blackman, Redford 2005. 
l'étain et peintes en polychromie - originaires des ateliers d'Italie du sud et de Sicile étaient commercialisées à Chypre. À Paphos, sur les sites de Fabrika, Saranda Kolones et Garrison's Camp, on trouve des exemplaires de proto-majolique des Pouilles, de Brindisi ou de Tavoliere ${ }^{34}$. À Famagouste, c'est une proto-majolique de type Gela Ware, provenant des ateliers de Sicile, qui a été découverte dans la vieille ville ${ }^{35}$.

Au début du règne des Lusignan, la composition du vaisselier à Chypre, tel qu'il peut être reconstitué à partir de l'exploration archéologique, a évolué vers une plus grande diversité. Les céramiques communes, fabriquées localement, côtoyaient toujours des importations de Beyrouth et la vaisselle fine provenaient de l'Empire byzantin et de Syrie mais aussi, et c'était nouveau, de Cilicie, des Pouilles et de Sicile. Toutefois l'arrivée des Francs à Chypre n'a pas été sans conséquence sur la fabrication de céramique. En effet, dans la région de Paphos, au milieu du XIII ${ }^{\mathrm{e}}$ siècle, dans des ateliers, dont les vestiges partiels ont été mis au jour à Lemba et à Paphos ${ }^{36}$ sur le site de Fabrika ${ }^{37}$, des potiers réalisaient de la vaisselle de table glaçurée, selon un savoir-faire qu'ils partageaient avec leurs confrères byzantins ou levantins. Ils fabriquaient des coupes, des plats et des cruches tournés dans une pâte argileuse, fine, rouge foncé et très dure, qu'ils peignaient d'abord à l'engobe de spirales ou de lignes ondulées sous une glaçure plombifère brillante, qu'ils couvraient plus simplement d'une glaçure monochrome sur engobe, ou qu'ils incisaient plus ou moins finement. Sur ces décors sgraffito étaient ensuite appliquées des glaçures plombifères monochromes, incolores, très brillantes, parfois rehaussées de pigments colorés verts et ocre jaune. Ces premières productions de vaisselle de table à Chypre, créées apparemment ex-nihilo, sont généralement considérées comme une sorte de variante locale des fabrications byzantines, constituant en quelque sorte une branche méridionale de cette activité artisanale. Il est vrai que, dans l'Empire byzantin disloqué après la quatrième Croisade, les ateliers de potiers se sont multipliés : de nouvelles officines ont ouvert dans le Despotat d'Épire, l'Empire latin, l'Empire de Nicée et l'Empire de Trébizonde ${ }^{38}$. Cependant la question qui se pose est de savoir pour quelles raisons des potiers byzantins ont choisi de délocaliser leur activité à Chypre, à une époque où l'île était entre les mains des Francs et alors que, pendant la seconde domination byzantine du $\mathrm{X}^{\mathrm{e}}$ à la fin du XII siècle, il n'y a eu aucune production de poterie glaçurée dans l'île. En l'absence d'élément de réponse, il paraît nécessaire de remettre en question la filiation supposée entre les ateliers byzantins et les premières officines de Chypre franque. Avec l'arrivée des Francs

34. Le matériel de la colline Fabrika, provenant des fouilles françaises dirigées par C. Balandier (université d'Avignon), est étudié par l'auteur : François 2011. Pour les autres sites, voir Giudice et al. 1996, p. 249, pl. XXXVIII, 4 ; Megaw 1972, p. 336, fig. F, 2, et fig. 30 ; Patitucci-Uggeri 1985, p. 385-386 et 388, fig. 23.

35. Mogabgab 1937-1939, p. 186, pl. XXXVI, 2.

36. Papanikola-Bakirtzi 1996, p. 55-137 ; Wartburg 1997b, p. 323-340.

37. À Kato Paphos, des surcuits et des pernettes ont notamment été découverts sur la colline Fabrika dans les fouilles australiennes et françaises : Cook, Green 2002, p. 413 ; François 2011.

38. François 2011. 
réfugiés de Terre sainte, Chypre s'était éloignée de l'influence de Constantinople pour s'inscrire davantage dans le Levant. Dans ce contexte, il n'est pas impossible que les premières productions de vaisselle fine de Paphos aient été réalisées par des potiers qui étaient en activité dans le royaume latin de Jérusalem, ou plus probablement dans la principauté d'Antioche, et qui ont accompagné les Francs dans leur repli sur Chypre. Les premières productions « franques doivent être considérées au regard de ce qui se faisait dans les États croisés du Levant au début du XIII ${ }^{\mathrm{e}}$ siècle. Les artisans de la terre travaillant dans le royaume latin de Jérusalem, mais aussi dans la principauté d'Antioche et le royaume d'Arménie-Cilicie, fabriquaient des céramiques en terre argileuse, engobées et glaçurées au plomb, qu'ils décoraient à l'engobe ou incisaient et couvraient de glaçures plombifères monochromes ou polychromes ${ }^{39}$. Une des formes les plus caractéristiques de leurs productions, en particulier des céramiques «d'Al-Mina », se retrouve parmi les vases issus des officines chypriotes ${ }^{40}$. Il s'agit de coupes à panse hémisphérique profonde terminée par un marli horizontal, souvent souligné par une arête à la jonction entre panse et lèvre (Fig. 1.6), et de coupelles profondes à panse carénée et marli large horizontal ou légèrement incliné. Remarquons que ces formes adoptées par les potiers de Paphos sont absentes du répertoire byzantin. De plus, comme nous le verrons, certains décors des céramiques «d'Al-Mina » se retrouvent sur les plats chypriotes. L'existence d'analogies morphologiques et ornementales fortes entre les céramiques de Paphos, au début du royaume des Lusignan, et les poteries réalisées dans la région d'Antioche et en Cilicie milite en faveur d'une migration d'artisans venus du Levant et qui ont accompagné les Francs dans leur retraite ${ }^{41}$. Comme d'autres artisans quittant les États croisés, ils sont venus exercer leur activité à Chypre en conservant leur savoir-faire. L'origine levantine des potiers de Paphos pourrait par ailleurs avoir facilité la commercialisation de leur production dans les États croisés avec lesquels ils maintenaient des liens ${ }^{42}$. En effet, on retrouve la trace des céramiques de Paphos sur de nombreux sites côtiers qui étaient occupés par les Francs - du nord au sud, Mersin en Cilicie ${ }^{43}$, Al-Mina dans le Hatay, Tripoli, Beyrouth, Acre, Sainte Marie-du-Carmel, Atlit, Yoqne'am, Césarée, al-Burj al-

39. Pour des exemples, voir Stern, Waksman 2003, p. 167-180.

40. Avissar, Stern 2005, p. 21, fig. 7, 9, 11 ; p. 23, fig. 8, 1, 1, et p. 55, fig. 22, 2-3 ; Pringle 1985, p. 178, fig. 3 : 20, p. 181, fig. 4, 21-22 ; Djobadze 1986, p. 186-198, fig. LX-LIV et LXVIII.

41. Les réfugiés qui ont migré vers Chypre au XIII ${ }^{\mathrm{e}}$ siècle étaient des Italiens - des Pisans et des Génois de Marqab, au sud de la principauté d'Antioche, se sont installés à Famagouste - et des chrétiens de Syrie. Quelques-uns étaient des artisans, d'autres des marchands : Coureas 2005, p. 128.

42. Sous la domination franque, les Arméniens étaient bien intégrés dans la société féodale. Les relations diplomatiques entre les Lusignan et le royaume de Petite Arménie étaient cordiales, et les échanges commerciaux entre la Cilicie et Chypre étaient denses : Otten-Froux 1996, p. 157-179.

43. Köroğlu 2007, p. 441-456, fig. 5. 
Ahmar, Arsuf ${ }^{44}$. Les fabrications de vaisselle fine chypriote n'étaient pas les seules à alimenter le commerce vers le Levant, puisque des marmites et des cruches modelées, fabriquées à Chypre, étaient également vendues sur les côtes proche-orientales. Leur présence, signalée à Acre, Atlit et Horbat 'Uza ${ }^{45}$, est d'autant plus troublante que ces poteries ne pouvaient sans doute pas rivaliser avec les céramiques culinaires levantines qui étaient glaçurées et donc nettement plus faciles à nettoyer. Ces relations fortes entre Chypre et le Proche-Orient sont confirmées par l'absence de poterie chypriote au Levant après la chute de Saint-Jean-d'Acre. Il faut souligner par ailleurs que les céramiques de Paphos n'ont pas été commercialisées dans l'Empire byzantin disloqué.

Si la diffusion des productions chypriotes au XIII ${ }^{\mathrm{e}}$ siècle a pu se faire à petite échelle, d'un rivage à l'autre, cette vaisselle a également alimenté le grand commerce alors aux mains des Italiens. L'implication des marines latines dans la distribution des poteries est illustrée par l'épave de Novy Svet découverte dans la baie de Soldaïa, au large de la Crimée. Il s'agit vraisemblablement d'un navire pisan qui, après une course poursuite à travers la mer Noire depuis Constantinople, a été incendié et coulé le 14 août 1277 par une galère génoise, en représailles de rixes qui avaient opposé des marins génois et pisans à Constantinople ${ }^{46}$. Ce navire renfermait dans ses cales : des Graffita arcaica tirrenica des ateliers de Savone et des Roulette Wares de Venise ; des amphores byzantines de type Günsenin 3 et 4, de la Glazed White Ware IV, des Zeuxippus Wares et des Aegean Wares ainsi que des céramiques de « Novy Svet» byzantines; des coupes des ateliers chypriotes de Paphos ; des céramiques «d'Al-Mina »; des marmites de Beyrouth et quelques céramiques seldjoukides d'Anatolie. Ces différentes sortes de vaisselle de table transportées par un navire italien à destination probable de Soldaïa, le centre de l'activité commerciale de Venise en Crimée, témoignent du rôle joué par les flottes italiennes dans la diffusion des productions des bassins occidentaux et orientaux de la Méditerranée au XIII ${ }^{\mathrm{e}}$ siècle. On retrouve des assemblages similaires sur les sites de consommation du ProcheOrient. Ces Frankish Assemblages, ainsi nommées par D. Pringle ${ }^{47}$, sont constitués : de céramiques culinaires glaçurées de Beyrouth ; de Gouged Wares, de vaisselle de table à pâte siliceuse et glaçure alcaline, et de Hand Made Geometric Painted Wares plus rustiques, de Syrie ayyoubide ; de coupes incisées fabriquées dans les ateliers francs de Paphos, mais aussi de marmites et de jarres modelées ; d'Aegean Wares et de Zeuxippus Wares byzantines ; et de productions d'Italie septentrionale et méridionale.

La présence de ces céramiques aux multiples origines et retrouvées associées en proportions variables sur les sites de Syrie-Palestine, est principalement caractéristique des lieux occupés par les Francs - de tels assemblages n'apparaissent pas dans les grandes

44. Avissar, Stern 2005, p. 57-62 ; Stern 2008, p. 455-469, table 1.

45. Stern 2008, p. 458-460, fig. 2, 11, 12.

46. Waksman, Teslenko, Zelenko 2009, p. 851-856.

47. Pringle 1982, p. 109 ; Pringle 1986, p. 451-475. 
villes d'Alep et de Damas ${ }^{48}$. Cependant, tout ou partie de ces céramiques étaient vendu sur les marchés d'Alexandrie et du Caire, hauts-lieux du commerce international ${ }^{49}$, et on les retrouve en quantité moindre dans le bassin occidental de Méditerranée ${ }^{50}$. Ces associations répétées de productions italiennes, byzantines, chypriotes et levantines constituaient, selon D. Pringle, les indices d'un réseau de distribution commun, peutêtre un commerce de cabotage alimenté par de grands centres qui servaient de relais dans la diffusion de la céramique. Ainsi les premières productions de vaisselle d'époque franque à Chypre ont très vite trouvé leur place parmi les grandes catégories de poterie qui alimentaient le marché de la vaisselle en Méditerranée.

$\mathrm{Au} \mathrm{XIV}$ e siècle, à Chypre, les productions locales de céramiques communes et de vaisselle de service se sont diversifiées. De nouveaux ateliers ont vu le jour. Pour autant l'île recevait toujours des produits étrangers qui ne se limitaient plus à la vaisselle de table, mais comptaient aussi des cruches pour rafraîchir l'eau et de la céramique architecturale venues de Syrie mamelouke. Si les fouilles livrent un bel échantillonnage de productions étrangères, quelques textes confirment la commercialisation de ces produits. Ainsi, à Chypre, dans la première moitié du XIV ${ }^{\mathrm{e}}$ siècle, les scodelle di terra faisaient partie de la longue liste de marchandises, d'un prix relativement important et sujettes à la taxation, qui étaient importées de Cilicie, de Syrie, de Turquie et de Rhodes ${ }^{51}$.

Deux fouilles permettent de dresser un inventaire des types de poteries employés dans l'île, dans la capitale à Nicosie au XIv ${ }^{\mathrm{e}}$ siècle ${ }^{52}$, et sur le territoire de Potamia-Agios Sozomenos mis en valeur par les Lusignan vers 1380. La découverte d'un puits, dégagé en 1988 au coin des rues Androcleous et Pindarou, au sud-est de la ville médiévale de Nicosie, à l'intérieur de l'enceinte des Lusignan, a fourni des marmites et des jattes à bec pincé, à pâte rouge, qui sont dans la tradition de ce qui se faisait à Chypre depuis le $\mathrm{XII}^{\mathrm{e}}$ siècle en ce qui concerne leurs formes, mais dans une version glaçurée au plomb ${ }^{53}$. Pour la même période, on en retrouve des exemplaires à Paphos, dans Icarus Street et sur la colline Fabrika ${ }^{54}$. Les cruches à pâte calcaire, ainsi qu'une amphorette jetées dans le puits ${ }^{55}$, ont sans doute pour origine un nouvel atelier implanté à Potamia. Une partie de la vaisselle de table découverte à Nicosie était importée, comme en témoignent un

48. Comme l'attestent les recherches récentes menées par l'auteur dans ces deux villes.

49. François 1999 (a) ; Scanlon 1967, p. 65-86 ; Scanlon 1971, p. 220-233 ; Scanlon 1984, p. 115-126.

50. Vallauri, Démians d'Archimbaud 2003, p. 137-152.

51. Balletto 1983, p. 138.

52. Flourentzos 1994. Une monnaie d'Hugues $\mathrm{I}^{\mathrm{er}}$ (1205-1218), trouvée au fond du puits, fournit un terminus post quem. On retrouve ces catégories dans le quartier de Chrysaliniotissa à Nicosie : Megaw 1951, p. 150, fig. 7 A.25.

53. Flourentzos 1994, pl. VII, XII, XXIII et XXIV, 52-54.

54. Gabrielli 2008, p. 442.

55. Flourentzos 1994, pl. VI,14-16, XXIV, 55, et XXV, 58. 
grand plat de céramique « d'Al-Mina », ainsi qu'une coupelle à pâte siliceuse peinte en noir et bleu sous glaçure alcaline incolore de Syrie ${ }^{56}$. Ces productions mameloukes sont également attestées dans le quartier de Chrysaliniotissa à Nicosie et dans la vieille ville de Famagouste ${ }^{57}$. Les importations orientales n'étaient d'ailleurs pas limitées à la vaisselle fine. En effet, des cruches pour rafraîchir l'eau, avec ou sans filtre, réalisées dans une pâte poreuse, claire, et incisés au peigne, caractéristiques des productions mameloukes de Syrie, ont été trouvées à Paphos ${ }^{58}$. Pour finir, diverses productions de vaisselle de table chypriotes faisaient partie du remplissage du puits. Une coupe de forme grossière, à pâte poreuse, sableuse, de couleur rosâtre, décorée au sgraffito, avec sur le bord à l'extérieur une couche d'engobe rouge, provient sans doute de l'atelier d'Enkomi ${ }^{59}$. Cette officine était située à une vingtaine de kilomètres au nord de Famagouste mais on en sait peu de chose, sinon qu'elle fonctionnait au XIV ${ }^{\mathrm{e}}$ siècle ${ }^{60}$. Sa production reste dans la tradition des fabrications de Paphos ${ }^{61}$. Enfin il n'est pas exclu que des coupes à pâte rose, de type Plain Glazed Wares, et des grands plats incisés peints polychromes soient des représentants d'ateliers implantés à Nicosie, dont l'existence est attestée par divers vestiges ${ }^{62}$. En effet, en 1947, à l'occasion de fouilles de sauvetage ouvertes au sud-est de la ville médiévale, à proximité des douves creusées au pied de la muraille vénitienne, plusieurs fours de potiers ont été repérés ${ }^{63}$. Dans le même quartier, plus récemment, des pernettes pour l'enfournement des poteries ont été trouvées dans la fouille de sauvetage du Palaion Demarcheion ${ }^{64}$. Pour autant, les productions de Nicosie sont encore mal connues.

L'exploration archéologique du territoire de Potamia-Agios Sozomenos, à $25 \mathrm{~km}$ au sud-est de Nicosie, a livré un ensemble de poterie daté de la fin $\mathrm{XIV}^{\mathrm{e}}-\mathrm{XV}^{\mathrm{e}}$ siècle ${ }^{65}$. Ce territoire a été mis en valeur à l'époque des Lusignan. Dans le derniers tiers du XIV ${ }^{\mathrm{e}}$ siècle, à Potamia, Jacques II ou Pierre I ${ }^{\mathrm{er}}$ a entrepris la construction d'un manoir conçu

56. Flourentzos 1994, pl. XXVI, 60, p. 16, fig. 4.

57. Megaw 1951, p. 148 ; Mogabgab 1937-1939, pl. XXXVI, 1 et 6.

58. François 2011, pl. 2, 5-7.

59. Flourentzos 1994, pl. IX.

60. Autour de l'église Agios Iakovos, en ruines, une pernette et deux vases sgraffito rejetés en cours de fabrication témoignent de l'existence d'un atelier dans les environs. La découverte, dans les années 1950, de vestiges de fours, de pernettes et d'un dépotoir d'atelier confirme bien l'existence d'une production locale qui reste mal connue : Papanikola-Bakirtzi 1989, p. 233-246.

61. Exemples dans Durand, Giovannoni 2012, p. 316-317 nos 139-140.

62. Flourentzos 1994, pl. XIII-XV, XX et XII, 42-43.

63. Information tirée du rapport de fouille rédigé par Megaw. Flourentzos 1994, p. 2-3.I.

64. Violaris 2004, p. 73, p. 80, fig. 18.

65. Programme de recherches intitulé La constitution des paysages en Orient médiéval : Potamia Agios/Sozomenos, qui a débuté en 2000 sous l'égide de l'EfA, en collaboration avec le LA3M et le Département d'histoire et d'archéologie de l'Université de Chypre. Ce programme était codirigé par N. Lécuyer, D. Michaelidès et G. Grivaud. Voir Lécuyer 2004, p. 11-29. 
comme une résidence de villégiature pour la famille royale installée à Nicosie ${ }^{66}$. Dans le village d'Agios Sozomenos, au nord du manoir, des églises et la découverte d'un trésor monétaire, datable entre 1368 et 1373, attestent l'existence d'un habitat dans ce secteur à la fin du XIV ${ }^{\mathrm{e}}$ siècle. Le matériel découvert en prospection, dans les sondages ouverts au manoir, ainsi que dans la fouille d'un moulin à eau ont permis d'élargir le répertoire morphologique des céramiques à pâte rouge modelée, vraisemblablement fabriquées à Kornos ou à Phoini ${ }^{67}$. Les grosses formes pour la conservation des liquides n'ont pas de revêtement (Fig. 2.2). La céramique culinaire est variée dans ses formes (Fig. 2.1). La glaçure est présente sur la plupart des vases allant au feu. Cette production en argile rouge lie-de-vin, particulièrement typique, est ornée avec beaucoup de soin : on trouve des cordons digités ou découpés, des pastilles appliquées, des répétitions d'impressions réalisées à la roulette, au peigne, ou avec des tiges creuses juxtaposées dans des jeux de compositions complexes d'ondes, de lignes, de rouelles, d'oculi, de hachures. Sur le site de Potamia, la prospection a livré les indices d'un artisanat de la terre pour des céramiques communes uniquement : des cruches, des jarres (Fig. 2.3) et des godets de noria réalisés en pâte calcaire, claire ${ }^{68}$. L'usage de vases à liquide en pâte calcaire est reconnu à Agios Sozomenos, comme le montre une petite cruche à bec pincé qui contenait le trésor de monnaies d'argent datées du dernier tiers du XIv e siècle. Sur la panse d'une cruche à pâte claire, un blason franc a été incisé avant cuisson (Fig. 2.4). Enfin, les fouilles du manoir royal ont livré 43 fragments de carreaux ornés de rinceaux végétaux et floraux finement dessinés au bleu de cobalt sous une glaçure alcaline ${ }^{69}$. Ils servaient de revêtement mural à une ou plusieurs pièces de cette résidence. Exceptionnels par leur petit module, ces carreaux de revêtement à pâte siliceuse seraient les plus anciens exemplaires des productions syriennes et constituent les seuls témoins d'un décor précieux, conçu à l'origine de la construction du manoir.

Dans la première moitié du $\mathrm{XIV}^{\mathrm{e}}$ siècle, les ateliers de la région de Paphos ont poursuivi leur activité, tandis que ceux d'Enkomi et probablement Nicosie, sans doute un peu marginaux, ont vu le jour. Leur activité a cessé à la fin du XIV siècle. C'est alors qu'un centre de fabrication important a pris le relais à Lapithos, sur la côte nord de l'île, un peu à l'ouest du port de Kyrénia. Une prospection menée par le Département des Antiquités chypriotes, ainsi que des fouilles de sauvetage ouvertes dans le quartier d'Agios Loukas, ont fourni une quantité impressionnante de déchets de cuisson et de pernettes, qui témoigne d'une intense activité de production dans ce village.

\section{3. À l'époque vénitienne : italianisation du vaisselier}

En 1474, Chypre a été intégrée au domaine colonial de Venise. La domination vénitienne a entraîné une italianisation progressive de la société. Elle s'est manifestée

66. Lécuyer 2006, p. 241-256.

67. François, Vallauri 2001, p. 530-534, fig. 3-5 ; Vallauri 2004, p. 225-226 et 233, fig. 5.

68. François, Vallauri 2001, p. 528-530, fig. 2, 21 ; Vallauri 2004, p. 232, fig. 1, 2.

69. Vallauri 2004, p. 227-228 et 235, fig. 10-11.. 
dans divers domaines. Les élites insulaires ont peu à peu adopté des modes culturelles italiennes ; elles ont envoyé leurs enfants étudier dans les universités d'Italie, en particulier à Padoue ; elles ont fréquenté les grandes villes du nord de la péninsule, alors que des citoyens de la Sérénissime sont venus s'installer à Chypre ${ }^{70}$. Peu à peu, la culture de la Renaissance italienne a pénétré dans la vie quotidienne des habitants de l'île et a influencé l'art insulaire : un style composite «italo-byzantin »s'est développé dans le domaine de la peinture d'icônes et de fresques. Cette italianisation s'est également manifestée dans le domaine de la vaisselle de terre, qu'il s'agisse des productions réalisées localement ou des céramiques étrangères commercialisées sur les marchés de Chypre.

Dans un tel contexte, l'essentiel des céramiques importées provenait d'Italie, d'ateliers vénéto-padans, ligures et toscans. Des graffita tarda, des céramiques haut de gamme incisées sous glaçure plombifère, fabriquées en Italie du Nord aux $\mathrm{Xv}^{\mathrm{e}}$ et $\mathrm{XVI}^{\mathrm{e}}$ siècles, apparaissent sur de nombreux sites, dans des contextes variés. À Potamia, un tesson avec la représentation d'un homme enturbanné figuré de profil, rehaussé de pigments brunâtres et bleus, est sans doute originaire de Lombardie ou de Vénétie ${ }^{71}$, et une belle coupe incisée, polychrome, de Padoue, a été trouvée sur le sol du moulin de Paléomylos ${ }^{72}$. Des coupelles de même type ont été retrouvées à Paphos, sur la colline Fabrika $^{73}$ (Fig. 2.5) et à Garrison's Camp ${ }^{74}$. À Nicosie, des coupes ornées de rinceaux végétaux excisés, d'animaux et de portraits incisés sont attribuées aux ateliers de Bologne pour le $\mathrm{Xv}^{\mathrm{e}}$ siècle ${ }^{75}$. À Famagouste, un fragment avec un soldat casqué est identifié comme un exemplaire de la production padane du Xvi siècle ${ }^{76}$. À Palaipaphos, un angelot représenté sur fond de décor architectural antique typique de la Renaissance orne une coupe, datée entre le $\mathrm{Xv}^{\mathrm{e}}$ et le début $\mathrm{du} \mathrm{XvI}^{\mathrm{e}}$, qui proviendrait des officines de Padoue ou d'Aquilée ${ }^{77}$; sur ce site des coupelles sont ornées de rinceaux végétaux et d'une grande fleur de même type que celles trouvées dans une tombe proche de l'église de la Chrysanayiotissa près d'Épiskopi ${ }^{78}$. Enfin, dans le musée du manoir royal des Lusignan, à Kouklia, une coupelle décorée d'un lièvre incisé sous rehauts polychromes, datée de la deuxième moitié du Xv ${ }^{\mathrm{e}}$ siècle, proviendrait d'Émilie ${ }^{79}$. D'autres productions d'Italie

70. Grivaud 2012, p. 328-333.

71. Francois, Vallauri 2001, p. 535, fig. 6, 17, p. 537 ; Nepoti 1991, pl. XXXIV.

72. Vallauri 2004, p. 228 et 235, fig. 12.

73. Cook, Green 2002, p. 416, 422, fig. 2, 16-18, et p. 426, pl. 11, 17 ; François 2011, pl. $3,1$.

74. Giudice et al. 1993, p. 327, fig. 15, 5, pl. XC, 2-5.

75. Du Plat Taylor, Megaw 1951, p. 12, pl. IX, 2 et 4, 27.

76. Mogabgab 1937-1939, p. 190, pl. XXXVI, 4.

77. Wartburg 1997a, p. 194, pl. XXI, 6-7.

78. Wartburg 1998, p. 162, 71-72 ; Du Plat Taylor 1938, fig. 15.

79. Pour des exemples proches, voir Nepoti 1991, p. 191 n $^{\circ} 17,193$ n $^{\circ} 23$ et 195, n 27 ; Siviero 1986, p. 29-41. 
septentrionale apparaissent également parmi les importations recueillies à Potamia ${ }^{80}$ : un fragment de panse d'une grande coupe, orné à l'extérieur de trois ressauts imprimés à la roulette, est semblable à un vase complet de Paphos ${ }^{81}$, ainsi qu'aux nombreuses coupes du sanctuaire d'Aphrodite de Palaipaphos ${ }^{82}$, et à quelques coupes de Fabrika à Paphos ${ }^{83}$. Des coupes dites a graffita monochroma, en pâte orange, recouverte d'engobe et de glaçure jaune ou orangée, qui proviennent de Ligurie, probablement des officines de Savone, étaient utilisées à Potamia comme à Kouklia ${ }^{84}$. On peut également restituer aux ateliers ligures ou padouans quelques fragments d'assiettes, avec ou sans marli, en faïence, ornées d'un décor floral très fin d'inspiration orientale, dit calligrafico a volute tipo $C$ peint en bleu foncé sur un fond blanc ou bleu pâle (Fig. 2 .6-7). Cette production datée $\mathrm{du} \mathrm{XVI}{ }^{\mathrm{e}}$ siècle est reconnue à Potamia ${ }^{85}$, à Nicosie ${ }^{86}$, à Paphos ${ }^{87}$ et parmi les collections du musée de Limassol. Elle côtoie des exemplaires de majolique ligure a berretino ${ }^{88}$. Diverses productions de Toscane se retrouvent aussi sur les sites chypriotes. À Potamia, des cruches pansues en faïence, peintes en polychromie, proviennent des officines de Montelupo ${ }^{89}$, ainsi que d'autres cruches découvertes à Agia Photou à Famagouste ${ }^{90}$, et sur les sites de Garrison's Camp et Fabrika à Paphos ${ }^{91}$. Une coupe à décor ovali e rombi de Montelupo a également été découverte au moulin de Paléomylos à Potamia.

La production locale de vaisselle de table a, elle aussi, été touchée par la mode italienne comme en témoignent les productions d'un atelier en activité à Famagouste entre la fin $d u x^{e}$ et le deuxième tiers du $\mathrm{XvI}^{\mathrm{e}}$ siècle. En effet, comme l'ont montré de récentes découvertes, les potiers de Famagouste réalisaient des coupes d'un style proche des céramiques vénéto-padanes, mais de moins bonne qualité ${ }^{92}$. Les traces de cette activité céramique - des pernettes, des nodules de cuivre utilisés pour la coloration en vert du vernis au plomb, une brique vitrifiée de la paroi d'un four, des moutons, des ratés de cuisson, ainsi que des biscuits - ont été observées sur un terrain au nord-ouest

80. François, Vallauri 2001, p. 535, fig. 6, 21.

81. Giudice et al. 1993, p. 290 et 324, fig. 12, 14-15, et fig. 13, 1, pl. LXXXV, 2.

82. Wartburg 1998, p. 161, fig. 64-67.

83. Cook, Green 2002, p. 417 et 424, fig. 2, 19.

84. Francois, Vallauri 2001, p. 535, fig. 6, 19-22 ; von Wartburg 1998, p. 160-161, fig. 81, 60.

85. François, Vallauri 2001, p. 539.

86. Du Plat Taylor, Megaw 1951, pl. IX, 4, 24, 29.

87. François 2011, pl. 3, 3.

88. Vallauri 2004, p. 235, fig. 13.

89. Vallauri 2004, p. 235, fig. 13.

90. Mogabgab 1937-1939, p. 190, pl. XXXVI, 7.

91. Giudice et al. 1993, p. 295 et 327, fig.15, 7, pl. XC, 6-7 ; François 2011, pl. 3, 4-5.

92. La forme la plus caractéristique correspond à une coupe à panse carénée soulignée par un ressaut et des cannelures. 
de la vieille ville de Famagouste, autour des églises de Sainte-Marie du Carmel et des Arméniens, en face du bastion San Luca, ainsi que sur la rampe d'accès au bastion Martinengo ${ }^{93}$. Cette zone n'était pas occupée par des maisons à l'époque vénitienne, mais se trouvait en périphérie des quartiers d'habitation et à proximité de l'enceinte. Les céramiques à pâte calcaire, de couleur crème ou rose, glaçurées en vert ou jaune, fabriquées dans cette officine, proches par leur style des productions des régions de Venise et de Padoue, et mêlées à des fragments de faïences et de graffita d'Italie du Nord et de vaisselle de Lapithos, militent en faveur d'une production $\mathrm{au} \mathrm{XVI}^{\mathrm{e}}$ siècle. La fin de la période d'activité de l'atelier peut coïncider avec la construction en 1561, à l'angle nordouest de l'enceinte, du bastion pentagonal Martinengo qui a entraîné le réaménagement du secteur et, par conséquent, la destruction des structures de production. Compte tenu du renouveau de Famagouste à l'époque de l'administration vénitienne, il est vraisemblable que l'implantation d'ateliers de potiers était encouragée et favorisée par les nouveaux maîtres de la ville.

À cette époque, d'autres officines fonctionnaient encore dans l'île. Aux $\mathrm{xV}^{\mathrm{e}}$ et $\mathrm{XVI}^{\mathrm{e}}$ siècles, la fabrication dans le Troodos de céramiques communes à pâte rouge liede-vin se poursuivait en évoluant dans leurs formes ${ }^{94}$. À Potamia, des bords épais en bourrelet ou aplatis pourraient appartenir à des cols de jarres ou à des vases de stockage sphériques ${ }^{95}$. Ce type de pithari a été retrouvé, en place, dans la cour d'une maison à Kouklia datée par une monnaie de 1571. Cette grosse jarre appartient à la catégorie des céramiques modelées en pâte rouge à décor incisé, retrouvée en grand nombre avec des sgraffito tardifs de Lapithos ${ }^{96}$. On peut en observer quelques exemplaires complets dispersés dans les jardins de l'abbaye de Bellapaïs. Et curieusement, sur la partie supérieure de la panse de ces jarres, des blasons armoriés francs ont été incisés avant la cuisson. Des écus de type différent apparaissent sur le même objet. À Potamia, la fabrication des mêmes formes de céramiques à pâte calcaire a perduré avec peu d'évolution. Cependant, les décors peignés étaient plus complexes. Pour sa part, la production des ateliers de Lapithos a évolué vers une extrême simplification des décors ${ }^{97}$. Des coupes à panse hémisphérique sur pied annulaire bas, de type Green Painted Sgraffito Wares, réalisées à la fin $\mathrm{du} \mathrm{XV}^{\mathrm{e}}$-début $\mathrm{du} \mathrm{XVI}^{\mathrm{e}}$ siècle, portent désormais une ornementation très simple, incisée et peinte grossièrement. Les Green Painted Wares, dans la première moitié du Xvi siècle, apparaissent comme une forme décadente de la série précédente. Les décors peints en vert sont simplifiés à l'extrême.

93. François, Vallauri 2010, p. 295-310.

94. Gabrielli 2008, p. 428 et 430.

95. François, Vallauri 2001, p. 531 et 533, fig. 3, 1-4..

96. Maier 1979, p. 172, pl. XVI, 4 et XVII, 2.

97. Les ateliers de Paphos ont poursuivi leur activité, sans doute sans interruption, jusqu'en 1974 : Papademetriou 2005 ; Ionas 2000. 
Durant l'époque vénitienne, Chypre était renommée pour ses tissus - soie, lin, coton et laine - qu'elle exportait dans toute l'Europe et en particulier à Venise, pour ses ouvrages de broderie et pour son orfèvrerie qui connaissaient un nouvel essor. Pour sa part, l'artisanat de la terre avait beaucoup perdu de son originalité. Une partie des fabrications locales de vaisselle fine était désormais très nettement marquée par l'Italie à Famagouste, tandis qu'une autre partie, réalisée à Lapithos, s'était considérablement appauvrie d'un point de vue technique et décoratif. Dans cet atelier, les artisans en activité à l'époque vénitienne n'ont apparemment rien conservé du patrimoine franc, et à peu près tous les éléments morphologiques et ornementaux qui caractérisaient la vaisselle du XIV ${ }^{\mathrm{e}}$ et du début du $\mathrm{Xv}^{\mathrm{e}}$ siècle ont disparu. Ces productions locales bas de gamme ne pouvaient rivaliser avec les importations italiennes, les graffita aux décors figuratifs et zoomorphes sophistiqués, et les faïences peintes dans des couleurs chatoyantes.

On ne saurait terminer l'inventaire des centres de fabrications d'époque franque sans signaler encore que des pernettes et des déchets de cuisson ont été découverts à Soloi. Cependant le matériel associé à ces trouvailles n'a pas été étudié ${ }^{98}$. Par ailleurs, si des voyageurs, au milieu du $\mathrm{Xv}^{\mathrm{e}}$ siècle, rapportaient l'existence d'ateliers à Nicosie, l'archéologie n'en a pas encore livré les vestiges ${ }^{99}$. Enfin, comme l'a souligné M.-L. von Wartburg, il existe à Chypre une série de vases de type Polychrome Painted Ware typique des XIV ${ }^{\mathrm{e}}$ et $\mathrm{XV}^{\mathrm{e}}$ siècles, qui ne peut être attribuée aux ateliers déjà reconnus ${ }^{100}$. Elle formule l'hypothèse, sur la base de l'observation de la pâte, que ces céramiques pourraient avoir été fabriquées au nord de l'île, peut-être à Nicosie, Soloi ou Lapithos, ou dans d'autres centres producteurs qui restent à découvrir ${ }^{101}$.

\section{La vaisselle de terre comme témoin de «l'identité franque »}

Dans un tel cadre, quelle définition donner à la « vaisselle franque » à Chypre ? Cette céramique est habituellement considérée comme un tout. Il semble en fait qu'il faille différencier par période les coupes et autres coupelles réalisées dans le royaume des Lusignan et qui relèvent de cultures assez différentes. L'isolement des types de production selon qu'ils ont été réalisés au milieu du XIII ${ }^{\mathrm{e}}$, ou aux $\mathrm{XIV}^{\mathrm{e}}$ et $\mathrm{XV}^{\mathrm{e}}$ siècles, peut mettre en évidence quelques aspects de l'identité franque sur la vaisselle de terre.

\section{Au XIII siècle à Paphos : version insulaire des productions croisées de la principauté d'Antioche et répertoire composite}

Les premières céramiques à glaçure réalisées dans l'île sous le règne des Lusignan ont été fabriquées, au milieu du XIII siècle, dans la région de Paphos, où il semble que

98. Papanikola-Bakirtzi 1996, p. 211-212.

99. Violaris 2004, p. 73, note 16.

100. Wartburg 2007, p. 424.

101. Sur ce dernier site, les vestiges d'une production locale sont attribués aux $\mathrm{XV}^{\mathrm{e}}$ et $\mathrm{XVI}^{\mathrm{e}}$ siècles,

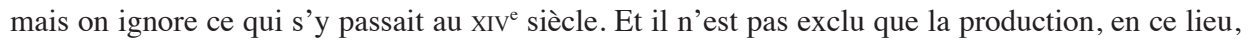
ait débuté plus tôt que ce qui en est dit. 
plusieurs ateliers se sont installés. Une partie de cette production, celle qui est incisée et rehaussée de pigments colorés appliqués en coulures, partage " un air de famille » avec les céramiques croisées réalisées dans la principauté d'Antioche et le royaume de la Petite Arménie ${ }^{102}$. Un examen des formes et des compositions décoratives révèle ces analogies. Les artisans de Paphos tournaient des coupes de toute forme ${ }^{103}$, dont un type est caractéristique de la vaisselle «d'Al-Mina »- une coupelle à panse hémisphérique profonde terminée par un large marli horizontal souvent souligné par une arête (Fig. 1.6). À ce premier indice d'une relation entre ces deux zones de production s'ajoutent divers éléments du répertoire décoratif tels que : des rinceaux fleuronnés organisés en boucle et rehaussés de pigments colorés ${ }^{104}$; une grande fleur à quatre pétales mise en valeur par des coulures vertes et ocre jaune et qui occupe la totalité de la surface intérieure ${ }^{105}$; un bandeau de cercles tangents assez grossièrement incisé sur le marli ${ }^{106}$; un gros nœud centré, à deux ou trois pointes, avec des rehauts polychromes ${ }^{107}$. En plus de ces motifs géométriques, le traitement en champlevé d'une face humaine « lunaire », placée dans un médaillon central, se retrouve aussi dans les deux productions. Des médaillons centraux hachurés, incisés ou champlevés, et considérés comme de possibles représentations de boucliers sarrasins, et des écus armoriés francs (Fig. 1.7), sont communs aux ateliers de Chypre et d'Al-Mina ${ }^{108}$. Des oiseaux et des poissons, qui avaient peut-être une charge symbolique qui nous échappe, apparaissent également sur des vases des deux origines ${ }^{109}$. $\mathrm{Si}$, au XIII ${ }^{\mathrm{e}}$ siècle, une partie du répertoire ornemental de la vaisselle de table relevait clairement des traditions décoratives développées dans les ateliers de la principauté d'Antioche et du royaume de Petite Arménie, une autre partie venait plutôt du monde byzantin. En effet, les artisans de Paphos fabriquaient des copies des Zeuxippus Wares byzantines, une vaisselle très populaire dans le bassin oriental de la Méditerranée, et qui était importée à Chypre ${ }^{110}$. En plus de ces imitations locales, les potiers empruntaient à cette catégorie de céramique de petits éléments décoratifs qui étaient placés dans des

102. Hobson 1932, p. 28-30, remarqua très tôt que les productions chypriotes entretenaient des liens stylistiques forts avec les céramiques d'Al-Mina. Quelques-unes de ces analogies ont été soulignées par Wartburg 2003, p. 163-165.

103. On peut en avoir un bon aperçu dans Papanikola-Bakirtzi 1996, p. 61, 86, 100-101, 116-117.

104. Riavez 2001, pl. 5, 3 et 9, 19 ; Papanikola-Bakirtzi 1996, pl. XXIII, 119-121.

105. Riavez 2001, pl. 5, 1 ; Papanikola-Bakirtzi 1996, pl. XIX, 96-99.

106. Riavez 2001, pl. 7, 13 et 8, 17 ; Papanikola-Bakirtzi 1996, pl. XXI, 108 et 110.

107. Riavez 2001, pl. 9, 20 ; Papanikola-Bakirtzi 1996, pl. VII, 34-35, XI, 56, XIV, 72 et XV, 77.

108. Lane 1937, pl. XXIII, 1, B ; Papanikola-Bakirtzi 1996, pl. XI, 60, XII, 65 et XVII, 86-87 ; Papanikola-Bakirtzi 2004, p. 66 ; Johns 1934, pl. LV, fig. 1-3 ; Papanikola-Bakirtzis 1996, pl. VIII, 41-42 ; Lane 1937, pl. XXIII, 2, A ; Papanikola-Bakirtzi 2004, p. 74-75.

109. Lane 1937, pl. XXII, 1, A, E ; Papanikola-Bakirtzi 1996, pl. VI, 25-29, VII, 30-31, X et XVI, 81-82.

110. Waksman, François 2004-2005, p. 665-668 et 715-717, fig. 18-20. 
bandeaux généralement incisés sur le bord des coupes. Des chevrons imbriqués, des sortes de doubles parenthèses, des X pointés, typiques de la Zeuxippus Ware, sont associés, à Paphos, à des décors nouveaux tels que des boucliers hachurés, des lions rampants, des poissons, des faces lunaires ${ }^{111}$.

En plus des blasons armoriés, éléments de l'identification statutaire qui faisaient partie intégrante de l'iconographie de la vaisselle croisée et chypriote, même s'ils ne peuvent être attribués aux grandes familles aristocratiques du Levant, on commence à trouver sur quelques pièces exceptionnelles des décors liés à l'univers culturel franc. La première de ces images est celle d'un fauconnier tenant un rapace perché sur un gantelet et entouré de deux autres oiseaux. Il s'agit de l'illustration d'une chasse pratiquée à l'aide d'oiseaux de proie, une activité partagée par l'aristocratie franque, en Occident comme en Orient, ainsi que par les élites byzantines et musulmanes ${ }^{112}$. Cependant, à Chypre, les chasses royales à l'épervier étaient fameuses et des chevaliers francs, devenus experts en la matière, ont rédigé des traités sur le sujet dans la seconde moitié du XIV siècle. Cette chasse était strictement réservée aux barons et aux chevaliers qui pouvaient ainsi passer plusieurs mois, installés à la campagne, à pourchasser toute sorte de gibier ${ }^{113}$. Au milieu du XIV ${ }^{\mathrm{e}}$ siècle, le voyageur Ludolf de Sudheim, impressionné par les moyens que les seigneurs francs consacraient à cette chasse à l'oiseau, attribuait au moindre noble de Chypre dix ou onze fauconniers ${ }^{114}$. Des danseuses et des musiciens incisés sur d'autres pièces de vaisselle renvoient également à de plaisantes activités de loisir, sans doute communes aux archontes byzantins et aux nobles francs ${ }^{115}$. Néanmoins la céramique fabriquée par les potiers en activité à Chypre, dès le milieu du XIII ${ }^{\mathrm{e}}$ siècle, n'était pas encore très marquée par l'univers culturel des Lusignan.

\section{Au XIV siècle : développement d'une iconographie franque originale et apparition du calice}

C'est seulement au XIV ${ }^{\mathrm{e}}$ siècle que sont apparus de véritables marqueurs identitaires francs sur la vaisselle réalisée dans les ateliers de Nicosie, d'Enkomi, ainsi que dans des centres qui ne sont pas encore localisés ${ }^{116}$, puis, à la fin de ce siècle, à Lapithos. Sur de

111. Pour des exemples voir notamment Papanikola-Bakirtzi 1996, pl. XII, 62, XIII, 66 et 68 , XVI, 81 et XVII, 86.

112. Il semble que l'art de dresser les faucons pour la chasse soit originaire du Moyen-Orient. Cette activité était très populaire à Byzance, où divers traités donnaient des conseils pour apprivoiser et dresser les animaux de proie. Dans les Assises du royaume de Chypre et de Jérusalem, plusieurs réglementations concernaient la possession des oiseaux de proie, qui représentait un privilège des classes économiquement fortes, ainsi qu'une marque de richesse et de puissance.

113. Papanikola-Bakirtzi 1996, pl. IX, 45.

114. Ludolphus de Sudheim 1884, p. 336.

115. Papanikola-Bakirtzi 1996, pl. XXI, 106, IX, 46-47, et XV, 73 ; Papanikola-Bakirtzi 2004, p. 51.

116. Comme je l'ai souligné, une série de coupes parmi lesquelles des pièces remarquables d'un point de vue iconographique ne peuvent être attribuées aux officines déjà connues. 
grands plats largement ouverts et sur des coupelles à panse cylindrique carénée, plus rarement sur des cruches pansues, est apparue une iconographie tout à fait particulière à Chypre et qui, il n'est pas inutile de le rappeler, n'entretenait aucun lien avec les céramiques réalisées en France à la même époque. Les images les plus remarquables sont des représentations soignées ou plus grossières de chevaliers francs armés de pied en cap. Capuchon de mailles sur lequel se place le heaume, haubert, chausse, épée et écu constituent leurs attributs les plus habituels sur les meilleurs exemples (Fig. 2.8). Sur d'autres coupes, un homme vêtu à la mode franque avec chausses et poulaines, parfois une épée à la main, enlace une femme richement parée portant un long voile de tête tombant jusqu'aux pieds ${ }^{117}$. Souvent, l'espace alentour est occupée par des blasons ou des oiseaux. Parfois, sur de grands plats, une ou deux femmes, magnifiquement costumées, tiennent entre leurs mains un hanap et une bouteille ${ }^{118}$. Les blasons armoriés, plus ou moins soigneusement tracés, font partie intégrante de l'iconographie de la vaisselle chypriote, même s'ils ne peuvent être déchiffrés ${ }^{119}$. On a voulu voir, dans le lion rampant incisé au fond d'une coupelle et entouré de blasons ${ }^{120}$, le lion des Lusignan qui apparaît au revers des monnaies d'Henri II (1285). Cependant le lion est la figure animalière la plus fréquemment représentée dans les armoiries médiévale : il peut donc appartenir à d'autres familles ${ }^{121}$.

Autre image relevant d'une tradition iconographique typiquement franque, la représentation de deux visages reliés entre eux par le cou : le premier est celui d'un vieillard barbu tandis que le second plus jeune est imberbe ${ }^{122}$. Cette composition n'est pas sans rappeler les grylles gothiques en vogue à la même époque en Occident ${ }^{123}$. Ces étranges créatures, des figures monstrueuses définies par des combinaisons de têtes ${ }^{124}$, ont pour origine la glyptique gréco-romaine. La composition monstrueuse du vase chypriote se rattache sans doute à la tradition romaine de Janvier à double face adoptée dans les calendriers gothiques, dans lesquels les visages du vieillard et du jeune homme

117. Papanikola-Bakirtzi 1997, p. 145 nos $78-79$.

118. Papanikola-Bakirtzi 1997, p. 143 n 75 ; Flourentzos 1994, p. 27, 28, pl. XIV-XV. Ces représentations peuvent être comparées aux buveurs dessinés sur la céramique de la principauté d'Antioche et de Cilicie : Wartburg 2001, p. 457-465 ; Redford 2004, p. 282-312.

119. Papanikola-Bakirtzi 2004, p. 78-79.

120. Papanikola-Bakirtzi 1997, p. 149 n 86.

121. Sur les écus des Lusignan voir Durand, Giovannoni 2012, p. 238.

122. Papanikola-Bakirtzi 1997, p. $148 \mathrm{n}^{\circ} 84$.

123. Les grylles, d'après un texte de Pline l'Ancien relatif à la caricature d'un certain Gryllos (porcelet), servent d'abord à désigner le genre satirique de la peinture à forte déformation, puis le nom finit par être appliqué à la glyptique représentant des êtres dont le corps est composé de têtes : Baltrusaitis 1993,p. 20.

124. Pour de nombreux exemples, voir Baltrusaitis 1993, p. 11-56. 
font référence au Passé et au Futur ${ }^{125}$. Peut-être est-ce ici une évocation du roi Janus de Chypre (1398-1432) ?

À la fin du XIV $\mathrm{XV}^{\mathrm{e}}$ siècle, les potiers de Lapithos ont commencé à tourner des objets d'une forme nouvelle et jusqu'alors inconnue en Méditerranée orientale : le calice. Il s'agit d'une coupelle d'un petit diamètre à l'ouverture, avec une panse cylindrique à carène basse ou plus rarement une panse hémisphérique, montée sur un piédouche plus ou moins élancé ${ }^{126}$. Cette base haute est parfois décorée de bandeaux appliqués et découpés, ou soulignée par plusieurs anneaux en relief, repoussés. À n'en pas douter, ces objets reprennent la forme de calices de métal précieux, sans doute employés à la cour, mais qui n'ont pas été retrouvés dans l'île. La surface extérieure des calices de Lapithos est ornée de décors géométriques organisés en bandeaux, tandis qu'on retrouve, à l'intérieur, des chevaliers et leurs dames, rendus le plus souvent de façon très schématique ${ }^{127}$. Cette simplification du décor, qui commence dès la fin du XIV ${ }^{\mathrm{e}}$, ira s'accentuant au cours du $\mathrm{XV}^{\mathrm{e}}$ siècle.

Ces représentations répondaient peut-être à la volonté des nobles francs établis dans l'île d'illustrer et de mettre en scène les valeurs fondamentales de leur culture, en l'occurrence l'éthique chevaleresque magnifiée dans la littérature épique diffusée à Chypre à cette époque. C'est ainsi que certaines images peuvent être interprétées en fonction de ces textes. La culture des Lusignan était profondément imprégnée de la tradition importée du royaume de France et des États latins de Terre Sainte. L'élément franc de la société chypriote restait fidèle à une culture de cour et les valeurs et les attitudes de la noblesse étaient exhibées de manières semblables à celles de leur pays d'origine. La classe chevaleresque de Chypre était en partie tournée vers l'Occident. Elle y trouvait son inspiration dans le domaine du comportement, du mode de vie et de l'éthique ${ }^{128}$. Des joutes, des banquets et des fêtes, mais aussi l'emploi du français comme langue commune et la circulation des livres contribuaient au développement d'une conscience de classe ${ }^{129}$. Des œuvres littéraires venues de France, rédigées en prose ou en vers, circulaient en Terre Sainte et à Chypre et étaient lues souvent à haute voix ${ }^{130}$. Dans la littérature lyrique,épique et courtoise, les chevaliers reconnaissaient le langage, les images et les symboles qui leurs étaient familiers ${ }^{131}$. Plusieurs œuvres majeures circulaient. La première d'entre elles, en partie écrite par Chrétien de Troyes au XII ${ }^{\mathrm{e}}$ siècle, est la matière de Bretagne appelée aussi Cycle d'Arthur ou Cycle de la Table Ronde, qui relate les aventures d'Arthur, roi

125. Baltrusaitis 1993, p. 39-40.

126. Papanikola-Bakirtzi 1996, pl. X-XI.

127. À titre d'exemple, on comparera la jeune femme au hanap du plat de Nicosie avec celle figurée sur un calice de Lapithos : Papanikola-Bakirtzi 2004, p. 53 n 3.

128. Jacoby 1986, p. 158 ; Grivaud 2009, p. 175.

129. Jacoby 1984, p. 619 ; Grivaud 2009, p. 77-91.

130. Stanesco 1998, p. 49 ; Ciggaar 1993, p. 89-97 ; Grivaud 2009, p. 61-63.

131. Jacoby 1986, p. 159. 
légendaire du Pays de Galles ${ }^{132}$. Ce premier texte a été complété par trois autres romans en prose : Tristan et Yseult, rédigé par Thomas vers 1174 ; Lancelot, composé entre 1215 et 1230, qui est parvenu en Orient entre cette dernière date et 1265 ; Palamède, connu au Levant en $1286{ }^{133}$. Autre ouvrage d'importance, le Roman de Troie qui fait partie de la matière de Rome ${ }^{134}$. Il met en œuvre des sujets antiques conformes au goût du public chevaleresque en développant notamment les histoires d'amour de Jason et Médée, de Pâris et Hélène, de Troïlos et Briséis, d'Achille et Polyxène. Ce texte témoigne d'une «médiévalisation » de l'Antiquité ${ }^{135}$; les héros antiques portant l'habit de chevalier sont dévoués à leurs dames et combattent selon les codes d'honneur de la chevalerie. Le Roman d'Alexandre, un autre récit important relevant de la matière de Rome, a été composé par Alexandre de Paris vers 1180, à partir de textes antérieurs et complété par de nombreux continuateurs ${ }^{136}$. Dans cette œuvre en vers, qui tient à la fois de la chanson de geste et du poème hagiographique, le héros macédonien est présenté comme un «Français à la mode de Grèce » ${ }^{137}$. Modèle de noblesse généreuse et d'ardeur juvénile, de courtoisie et de sagesse, Alexandre le Grand est l'archétype du parfait chevalier médiéval qui se reconnaissait en particulier dans ses aventures en Orient. À Chypre, le Roman d'Alexandre semble connu dès le début du XIII" siècle ${ }^{138}$.

Tous ces ouvrages appartenaient à la grande tradition de la littérature romanesque qui transmettait l'éthique de la noblesse, le comportement courtois et la romance. Les chevaliers arthuriens et les héros antiques à la mode médiévale apparaissaient comme une communauté dont les valeurs pouvaient être partagées par les chevaliers francs et servaient de référence aux participants des joutes - des compétitions strictement réglées suivies de danses et de banquets remplis de «fantaisie héroïque ». Des épisodes de la Table Ronde et du Roman de Troie ont fait l'objet de mises en scène plus ou moins libres, dans le cadre d'interprétations théâtrales jouées par les chevaliers. Le chroniqueur Philippe de Novare rapporte qu'en 1223, lors de l'adoubement des fils de Jean I'r d'Ibelin, seigneur de Beyrouth, une fête somptueuse a été organisée à Chypre combinant joutes, festins, danses et divertissements en relation avec les Aventures de Bretagne ${ }^{139}$. La même chronique évoque les réjouissances splendides qui se sont déroulées en 1286 à Acre lors

132. Jacoby 1984, p. 627 ; Stanesco 1998, p. 66-82.

133. Raynaud de Lage 1976, p. 127-160.

134. Récit lyrique en vers composé vers 1165 par Benoît de Sainte-Maure, qui a été rendu plus accessible aux Français et adapté au goût des contemporains grâce à une traduction en prose dans la deuxième moitié du XIII ${ }^{\mathrm{e}}$ siècle : Woledge 1953, p. 313-324 ; Stanesco 1998, p. 58-60.

135. Raynaud de Lage 1976, p. 127-160.

136. Stanesco 1998, p. 60-62.

137. Gosman 1997.

138. Jacoby 1984, p. 625-626 et 642 .

139. Filippo da Novara, 16 (112) ; Loomis 1959, p. 553-554 ; Jacoby 1984, p. 627 ; Grivaud 2009, p. 175. 
du couronnement d'Henri II de Chypre comme roi de Jérusalem et d'Acre - pendant quinze jours, la noblesse du royaume, rassemblée dans l'auberge des Hospitaliers, a festoyé et a parodié en se travestissant divers épisodes de la Table Ronde et du Roman de Troie ${ }^{140}$. De grands seigneurs adoptaient les noms et les blasons de Gauvain, Lancelot ou Palamède et se déguisaient en femmes pour interpréter les Amazones de la Reine des Féminies, un épisode de l'Histoire universelle - une histoire du monde de la Création à la prise de pouvoir de Jules César, très populaire au Moyen Âge.

Pour les Francs, en Méditerranée orientale, ces festivités étaient un moyen de manifester une identification collective et de retrouver à travers elles leur propre image ${ }^{141}$. Acteurs et auditoire formaient un groupe social homogène qui consacrait ainsi son unité. Mais au-delà du comportement courtois, c'était à une situation politique que faisaient aussi référence ces ouvrages qui pouvaient être lus comme des rappels des raisons de la présence occidentale en Orient. En effet, la plupart des romans en circulation en Terre Sainte exaltaient la juste guerre des chrétiens contre les musulmans - dans le Roman d'Alexandre par exemple, les Perses sont assimilés aux Sarrasins, tandis que les Grecs se rassemblent autour de l'oriflamme du roi de France.

C'est dans ce contexte que peuvent être interprétées quelques images dessinées sur des céramiques fabriquées à Chypre au XIV siècle. Les deux femmes enlacées incisées au fond des coupes pourraient être une évocation des Amazones de l'Histoire Universelle ${ }^{142}$. C'est au Roman de Troie et aux aventures de Jason que pourrait se rapporter la représentation d'un centaure-archer coiffé d'un heaume et saisi en pleine chasse ${ }^{143}$. En effet, Jason a eu pour précepteur le centaure Chiron qui lui apprit l'art de la chasse avec Artémis. Ses fonctions d'éducateur rappellent la présence, aux côtés des héros, de conseillers considérés dans les romans courtois comme des modèles de sagesse et de vertu. La représentation de saint Georges ${ }^{144}$ pourrait être liée aux poèmes épiques des Croisades et particulièrement à la Chanson de Jérusalem qui vante à maintes reprises les prodigieux exploits du saint guerrier, considéré par les Croisés comme leur patron et leur protecteur ${ }^{145}$. Les dessins de volatiles dans les productions chypriotes ne sont pas rares, mais l'association très fréquente d'un chevalier et d'un oiseau, ou d'un blason centré entouré de nombreux oiseaux, peut avoir au moins deux significations. La première serait une évocation de la chasse à l'oiseau très pratiquée dans l'île. La seconde pourrait avoir une valeur plus symbolique. Il est en effet possible que cette image renvoie au Vøu $d u$

140. Cronaca del Templare di Tiro, 203 (439).

141. Jacoby 1986, p. 166 et 176 ; Jacoby 1984, p. 634 ; Grivaud 2009, p. 159-160.

142. Karageorghis 1985, n 276 ; Flourentzos 1994, pl. XV ; Piltz 1996, p. 42 n 25 et $43 n^{\circ} 26$.

143. Papanikola-Bakirtzi 1997, p. 150 n 89.

144. Papanikola-Bakirtzi 1997, p. 149 nº 87.

145. La chanson d'Antioche, dans Croisades et pèlerinages, chant III, p. 49 et 61, chant VIII, p. 163 ; La conquête de Jérusalem, dans Croisades et pèlerinages, chant I, p. 191-192, chant VI, p. 282, chant VIII, p. 341. 
Faisan, un épisode du Roman d'Alexandre. Le roi de Macédoine, considéré comme le créateur de la chevalerie, aurait juré sur le faisan de partir à la conquête de l'Orient. Aussi, au Moyen Âge, faire vœu sur un faisan ou sur un autre oiseau était, croyait-on, une coutume chevaleresque datant d'Alexandre ${ }^{146}$. Dans le contexte chypriote de nos représentations, l'oiseau placé à côté du chevalier, qui n'est pas un élément décoratif de remplissage, rappelle peut-être le vœu fait par les Francs de délivrer les Lieux saints.

Ces images participaient sans doute de la même manière que la littérature, les spectacles et les chasses, à la diffusion des valeurs et du style de vie de la communauté latine établie sur l'île. En contribuant à la représentation d'images et de symboles qui devaient être pleinement intelligibles, peut-être offraient-elles ainsi une autre forme de communication à un public qui n'avait pas forcément accès aux autres expressions visuelles, telles que les miniatures ou les fresques. Il s'agissait, à travers cette iconographie, d'une sorte de transmission matérielle de valeurs et de signes que les hôtes, au cours des repas, pouvaient reconnaître comme autant de traits distinctifs de leur société, s'ils étaient Francs, ou de la société des puissants, s'ils avaient une autre origine. Dans le premier cas, les utilisateurs étaient capables d'apprécier ces images d'un point de vue symbolique. Elles participaient, de la même façon que les œuvres historiques ou légendaires, à la formation de la conscience du chevalier expatrié et du Croisé, leur rappelant à la fois leur origine culturelle et leur mission en Terre sainte. Dans le second cas, elles rappelaient aux dîneurs quel était l'univers des maîtres auxquels ils étaient soumis.

Il est très difficile de savoir à qui était destinée cette vaisselle de terre. À la cour des Lusignan, on utilisait sans aucun doute de la vaisselle de métal précieux comme c'était déjà le cas sous les Comnène ${ }^{147}$. Un témoignage du milieu du $\mathrm{Xv}^{\mathrm{e}}$ siècle nous renseigne sur ce point. Le navire sur lequel voyageait Georges Lengherand, receveur général de Hainaut parti en pèlerinage, s'est arrêté en juillet 1486 au port de Salines pour y attendre Florence Crispo, afin de la conduire à Nicosie auprès de sa fille Catherine Cornaro, la reine de Chypre. Peu avant son arrivée, dans une maison de campagne à une lieue du port, les gens de sa maison l'ont précédé en apportant des victuailles et « vaisselle d'argent pour cuisine aultrement pour le servir » ${ }^{148}$. Au XIV siècle, la richesse des couches supérieures de la population - les grands barons, mais aussi les commerçants

146. Les Vœux du Faisan, un récit en prose écrit par Olivier de la Marche à la cour de Bourgogne, évoquait encore cette pratique à la fin $\mathrm{du} \mathrm{XV}^{\mathrm{e}}$ siècle. Lors d'une fête organisée à Lille en février 1454, la haute noblesse de Bourgogne a promis de partir en croisade et a fait vœu sur le faisan. La formule répétée successivement par les nobles rassemblés était « Je fais vœu à Dieu mon Créateur, à la glorieuse Vierge Marie sa mère, aux dames et au faisan, que si... ». Les Vocux du Faisan, dans Régnier-Bohler 1995, p. 1131-1163.

147. La Chronique de la troisième Croisade évoque le trésor abandonné par Isaac Comnène lors de la conquête de Chypre par Richard Cœur de Lion. Il contenait entre autre des coupes et des plats en or, des plateaux et des pots en argent que Richard trouva aussi en abondance dans les villes et les forteresses de l'île : Chronicle of the Third Crusade, p. 195.

148. Grivaud 1990, p. 127-128. 
prospères de Famagouste - était fameuse ${ }^{149}$. Le luxe dans lequel vivaient les Francs de Chypre a été rapporté par de nombreux pèlerins. Selon Ludolph de Sudheim, au mariage de la fille d'un habitant de Famagouste « des chevaliers français qui nous accompagnaient estimèrent que la couronne de la mariée était plus précieuse que les joyaux de la couronne de France ». Il insiste sur la grande richesse d'une partie de la population, des grands marchands et même des prostituées ${ }^{150}$. Les princes, nobles, barons et chevaliers de Chypre, ainsi que les bourgeois et les marchands qui, selon lui, étaient les plus riches du monde, se contentaient-ils de ces modestes coupes de terre ? On peut vraiment en douter, d'autant qu'au XIV e siècle une culture de luxe s'est affirmée stimulant l'artisanat local célèbre notamment pour ses fabrications de tissus précieux. Ces pièces de vaisselle n'étaient pas employées par la classe dirigeante ; elles servaient peut-être sur les tables des artisans, des marchands et des officiers royaux ou seigneuriaux grecs, arméniens, syriens, italiens. Contrairement à ce qui est souvent écrit et répété, cette céramique glaçurée n'était pas une vaisselle de luxe et, bien qu'elle porte des images empruntées à l'univers chevaleresque franc, son emploi n'était sans doute pas limité aux Latins.

\section{3. À partir du $\mathrm{XV}^{e}$ siècle : disparition progressive des marqueurs identitaires francs sur la vaisselle}

$\mathrm{Au} \mathrm{Xv}^{\mathrm{e}}$ siècle, la structure de la noblesse a évolué : sur 200 familles dont le nom est mentionné dans la liste des fiefs, il n'y en avait plus que 20 qui appartenaient à la vieille noblesse franque ${ }^{151}$. La langue française avait presque totalement disparu, remplacée par le grec et l'italien. La richesse et l'aisance dans laquelle vivait une partie de la population s'étaient amenuisées. La mentalité a aussi évolué. La société franque était alors devenue une société chypriote qui avait perdu son caractère féodal et occidental, et dans laquelle les deux éléments principaux de la population, la composante grecque et la composante franque, se sont interpénétrés. Dans ce contexte, les marqueurs identitaires francs incisés sur la vaisselle se sont alors dilués peu à peu pour disparaître totalement au XVI ${ }^{\mathrm{e}}$ siècle.

$\mathrm{Au}$ cours du $\mathrm{Xv}^{\mathrm{e}}$ siècle en effet, la simplification du décor figuratif s'est accentuée. $\mathrm{Au}$ fond des coupes et des calices, dont la fabrication se maintenait dans les ateliers de Lapithos, les chevaliers, représentés frontalement, ont souvent perdu bras et/ou jambes, et leur habit était simplifié à l'extrême. Les blasons incisés d'un geste rapide n'évoquaient plus que très vaguement les écus francs ${ }^{152}$. Ces images, devenues des stéréotypes, ont progressivement disparu pour laisser la place à des coupes au décor géométrique très simple. Pour sa part, la production de vaisselle à Famagouste au $\mathrm{XVI}^{\mathrm{e}}$ siècle ne relevait plus de l'univers culturel franc, mais reflétait désormais le goût des nouveaux maîtres de

149. Rudt de Collenberg 1982, p. 75.

150. Ludolph de Sudheim, dans Croisades et pèlerinages, p. 1051-1052.

151. Rudt de Collenberg 1982, p. 76-78 et 80.

152. Papanikola-Bakirtzi 2004, p. 53 n 3 et 147 nos 93-94 ; Papanikola-Bakirtzis 1996, pl. LXI, 76-77 ; Papanikola-Bakirtzi 1997, p. 156 n 101. 
l'île, les Vénitiens. La production de vaisselle à Chypre semble bien refléter la nouvelle situation politique qui a changé dès le milieu du $\mathrm{Xv}^{\mathrm{e}}$ siècle.

\section{L'exception chypriote}

Chypre, grâce à sa situation géographique privilégiée - l'île se trouve à proximité des côtes de l'Asie Mineure, de la Syrie et du Liban, et à moins de $400 \mathrm{~km}$ de celles de l'Égypte - était au cœur des échanges entre le Proche-Orient chrétien et musulman et le monde byzantin. Au Moyen Âge, son histoire, son peuplement, son économie et sa culture ont donc été fortement marqués par ces facteurs géographiques. Dans ce lieu d'échange et de rencontre, la production et le commerce de la vaisselle se trouvaient à un carrefour des cultures méditerranéennes et proche-orientales. Elles témoignaient d'une réalité multiethnique et multiculturelle difficile à retracer aujourd'hui.

Si la domination latine s'est maintenue pendant trois siècles entiers à Chypre, l'expression de cette culture à travers la production de poterie n'a pas été la même d'un bout à l'autre de la période. Le premier point sur lequel il convient d'insister est l'absence d'une production locale de vaisselle de table pendant la seconde domination byzantine, du $\mathrm{IX}^{\mathrm{e}}$ à la fin du XII ${ }^{\mathrm{e}}$ siècle. La création d'une fabrication de céramique glaçurée destinée au service et à la présentation des mets sur la table a coïncidé avec l'installation des Lusignan à Chypre. Les caractéristiques techniques, morphologiques et décoratives de ces premières fabrications chypriotes au milieu du XIII ${ }^{\mathrm{e}}$ siècle permettent de croire que les potiers qui en étaient à l'origine sont venus de la principauté d'Antioche ou du royaume de Petite Cilicie. Installés à Paphos, ils ont continué à fabriquer ce qu'ils faisaient auparavant, tout en agrémentant parfois la production d'une pointe d'ornements byzantins pour réaliser une vaisselle tout à fait originale qui, à cette époque, n'était pas encore très marquée par l'univers culturel des Latins. Au XIV siècle, la production de céramique de table se nourrissait toujours de l'héritage levantin mais incorporait des apports gothiques mettant en scène l'éthique chevaleresque. Cette vaisselle, dont les décors s'inspiraient probablement de la littérature, était sans doute destinée à une clientèle d'artisans, de commerçants, de petits fonctionnaires qui pouvaient être d'une grande diversité d'origines, de langues et de confessions - aux lignages francs, anciennement installés dans les États latins et privés de leurs terres par la conquête musulmane, se mêlaient des Occidentaux fraîchement débarqués, des Grecs autochtones, la grande majorité de la population, des Syriens, des Arméniens et des marchands italiens et catalans.

Chypre était un laboratoire exceptionnel où se nouaient des relations culturelles complexes, qu'on ne retrouve pas dans d'autres territoires occupés par les Latins à la même époque. Lors de la quatrième Croisade, après la prise de Constantinople au début du XIII ${ }^{\mathrm{e}}$ siècle, les Latins ont partagé le territoire byzantin. Sur les débris de l'Empire, des principautés franques, dont la principauté d'Achaïe ou de Morée, ont vu le jour. Le Péloponnèse était ainsi passé sous la domination franque de Guillaume de Champlitte et de Geoffroy de Villehardouin. Cette implantation latine, numériquement faible, s'est appuyée sur une aristocratie grecque locale, mais restait complètement occidentale par 
son genre de vie ainsi que par sa structure féodale. Dans ce « morceau de France en territoire grec » ${ }^{153}$, la littérature courtoise circulait de la même façon qu'à Chypre et s'adressait au même public ${ }^{154}$. Dans cette vaste région, les ateliers de potiers de Corinthe, Argos et Sparte, en activité bien avant l'arrivée des Francs, ont poursuivi leur production sous la domination latine. Les conditions semblaient donc réunies pour une fabrication de céramique imprégnée, comme à Chypre, de la culture des nouveaux occupants. Or, la vaisselle de table mise au jour dans le Péloponnèse ne partage en rien les caractéristiques des productions chypriotes. Elle s'est maintenue dans la tradition byzantine et ne pouvait rivaliser avec les importations italiennes, nombreuses et variées ${ }^{155}$. Il n'existait pas, dans la principauté d'Achaïe, un type de vaisselle directement lié à la culture occidentale chevaleresque, alors que la structure de la société franque et son univers culturel étaient assez proches de ceux de la Chypre franque. Cette comparaison renforce le caractère exceptionnel des productions de poteries à Chypre.

Aix-Marseille Université, CNRS-LA3M, Aix-en-Provence

\section{BIBLIOGRAPHIE}

Avissar (M.), Stern (E.J.), 2005, Pottery of the Crusader, Ayyubid, and Mamluk Periods in Israel, Jérusalem.

BaKirtzis (Ch.) éd., 2003, Actes du VII ${ }^{e}$ Congrès International sur la Céramique Médiévale en Méditerranée, Thessalonique 11-16 octobre 1999, Athènes.

Balletto (L.), 1983, « Cipro nel "Manuale di Mercatura" di Francesco Balducci Pegolotti », Miscellanea di Studi Storici 2, Collana Storica di Fonti Studi 38, p. 137-148.

Baltrusaitis (J.), 1993, Le Moyen Âge fantastique, antiquités et exotisme dans l'art gothique, Paris [rééd. de 1955].

Blackman (M.J.), Redford (S.), 2005, « Neutron Activation Analysis of Medieval Ceramics from Kinet, Turkey, especially Port Saint Symeon Ware », Ancient Near Eastern Studies 42, p. 83-186.

Cheynet (J.-Cl.), 1993, « Chypre à la veille de la conquête franque ", dans C. Mutafian (éd.), Les Lusignans et l'Outre-mer (Actes du colloque de Poitiers-Lusignan 20-24 octobre 1993), Poitiers, p. 67-77.

CiggaAr (K.N.), 1993, « Le Royaume des Lusignan : terre de littérature et de traductions, échanges littéraires et culturels », dans C. Mutafian (éd.), Les Lusignans et l'Outremer (Actes du colloque de Poitiers-Lusignan 20-24 octobre 1993), Poitiers, p. 89-97.

Cook (H.K.A.), Green (J.R.), 2002, « Medieval Glazed wares from the theatre site at Nea Pafos, Cyprus: Preliminary Report », RDAC, p. 413-426.

Coureas (N.), 2005, « Economy », dans A. Nicolaou-Konnari, Ch. Schnabel (éd.), Cyprus, Society and Culture 1191-1374, Leyde et Boston, p. 103-156.

Chronicle of the Third Crusade, a Translation of Itinerarium Peregrinorum et Gesta Regis Ricardi, éd. H.J. Nicholson, Aldershot, 1997.

Croisades et pèlerinages, récits, chroniques et voyages en Terre Sainte XII ${ }^{e}-X V I^{e}$ siècles, éd. D. Régnier-Bohler, Paris, 1997.

153. Ostrogorsky 1983, p. 446.

154. Jacoby 1986, p. 158-186 ; Grivaud 2009, p. 175 et 177.

155. François 1997, p. 392-400. 
Cronaca del Templare di Tiro (1243-1314). La caduta degli Stati Crociati nel racconto di un testimone oculare, éd. L. Minervini, Naples, 2000 .

DJobadze (W.), 1986, Archaeological Investigations in the Region of West of Antioch-onthe-Orontes, Stuttgart.

Du Plat Taylor (J.), Megaw (A.H.S), 1951, « Early Glazed Pottery from Polis », RDAC 1937-1939, p. 77-93.

Durand (J.), 2012, « Chypre et les arts somptuaires byzantins », dans Durand, Giovannoni 2012, p. 150-155.

Durand (J.), Giovannoni (D.) dir., 2012, Chypre entre Byzance et l'Occident $\mathrm{IV}^{\mathrm{e}}-\mathrm{XVI}^{\mathrm{e}}$ siècles, Catalogue de l'exposition du Musée du Louvre, Paris.

FilipPo da Novara, Guerra di Federico II in Oriente (1223-1242), éd. S. Melani, Naples, 1994.

Flourentzos (P.), 1994, A Hoard of Medieval Antiquities from Nicosia, Nicosie.

François (V.), 1997, « Céramiques importées à Byzance : une quasi-absence », Byzantinoslavica 57, 2, p. 387-404.

FrançoIs (V.), 1999a, La céramique médiévale à Alexandrie. Contribution à l'histoire économique de la ville, Le Caire.

FrançoIs (V.), 1999b, «Une illustration des romans courtois. La vaisselle de table chypriote sous l'occupation franque », CCEC 29, p. 59-80.

FRANÇOIS (V.), 2011, « Rapport préliminaire sur les céramiques d'époques byzantine, franque et ottomane découvertes dans les fouilles françaises de la Fabrika à Paphos », RDAC, sous presse.

FrançoIs (V.), 2012, « Représenter le commerce de la poterie à Byzance », dans É. Malamut, M. Ouerfelli (dir.), Les échanges en Méditerranée médiévale, Aix-en-Provence, p. 33-55.

François (V.), Vallauri (L.), 2001, « Production et consommation de céramiques à Potamia (Chypre) de l'époque franque à l'époque ottomane », $B C H 125$, p. 523-546.
François (V.), Vallauri (L.), 2010, «Famagouste : indices d'une production de vaisselle à l'époque vénitienne », CCEC 40, p. 295-310.

François (V.), Nicolaïdis (A.), Vallauri (L.), Waksman (S.Y), 2003, « Premiers éléments pour une caractérisation des productions de Beyrouth entre domination franque et mamelouke ", dans Bakirtzis 2003, p. 325-340.

GABRIELI (R.S.), 2007, «A Region apart: Coarse Ware of Medieval and Ottoman Cyprus », dans B. Böehlendorf-Arslan, A.O. Uysal, J. Witte-Orr (éd.), Çanak, Late Antique and Medieval Pottery and Tiles in Mediterranean Archaeological Contexts, Byzas 7, p. 399-410.

Gabrieli (R.S.), 2008, « Towards a Chronology - the Medieval Coarse Ware from the Tomb in Icarus Street, Kato Pafos », RDAC, p. 423-454.

Gabrieli (R.S.), McCall (B.), Green (J.R), « Medieval Kitchenware from the Theatre Site at Nea Paphos », RDAC, p. 335-356.

La Géographie d'Edrisi, trad. P.-A. Jaubert, II, Paris, 1840, p. 130 [rééd. par H. Bresc \& A. Nef, Paris, 1999].

Giudice (F.) et al., 1993, « Paphos, Garrison's Camp. Campagna 1989», RDAC, p. 279-327.

Giudice (F.) et al., 1994, «Paphos, Garrison's Camp. Campagna $1990 », R D A C$, p. 215-268.

Giudice (F.) et al., 1996, « Paphos, Garrison's Camp. Campagna 1991», RDAC, p. 171-268.

Gosman (M.), 1997, La légende d'Alexandre le Grand dans la littérature française $d u X^{e}{ }^{e}$ siècle, Amsterdam.

Grivaud (G.), 1990, Excerpta Cypria Nova, Volume premier, Voyageurs occidentaux à Chypre au XV siècle, Nicosie.

Grivaud (G.), 1998, « Villages désertés à

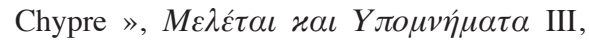
p. 1-604.

Grivaud (G.), 2009, Entrelacs chiprois. Essai sur les lettres et la vie intellectuelle dans le royaume de Chypre 1191-1570, Nicosie. 
Grivaud (G.), 2012, « Le doux royaume de Chypre (1192-1474) », dans Durand, Giovannoni 2012, p. 182-189.

Hild (F.), Hellenkemper (H.), 1990, Kilikien und Isaurien, 1. Teil, Vienne (Tabula Imperii Byzantini 5).

Hobson (R. L.), 1932, Guide to the Islamic Pottery, Oxford.

Ionas (I.), 2000, Traditional Pottery and Potters in Cyprus. The Disappearance of an Ancient Craft Industry in the 19th and 20th centuries, Aldershot.

JACOBY (D.), 1984, «La littérature française dans les états latins de la Méditerranée orientale à l'époque des croisades ", Essor et fortune de la chanson de geste dans l'Europe et l'Orient latin, Actes du IX Congrès International de la Société Rencesvals pour l'étude des épopées romanes (Padoue-Venise, 29 août-4 septembre 1982), t. II, Modène, p. 617-646 [repr. dans D. Jacoby, Studies on the Crusader States and the Venetian Expansion, Londres, 1989 , étude $n^{\circ}$ II].

JACOBY (D.), 1986, « Knightly Values and Class Consciousness in the Crusader States of the Eastern Mediterranean », Mediterranean Historical Review 1, p. 158-186 [repr. dans D. Jacoby, Studies on the Crusader States and the Venetian Expansion, Londres, 1989, étude $\left.\mathrm{n}^{\circ} \mathrm{I}\right]$.

Johns (C.N.), 1934, « Medieval Slip-Ware from Pilgrims'Castle, 'Atlit (1930-31) », QDAP 3, p. 136-144.

Karageorghis (V.), 1985, Ancient Cypriot Art in the Pierides Foundation Museum, Larnaca.

KÖROĞLu (G.), 2007, « Glazed Pottery from Cyprus and the Hatay-Çukurova Region in the Mersin-Yumuktepe Excavations », dans B. Böehlendorf-Arslan, A.O. Uysal, J. Witte-Orr (éd.), Çanak, Late Antique and Medieval Pottery and Tiles in Mediterranean Archaeological Contexts, Byzas 7, p. 441-456.

Lane (A.), 1937, « Medieval finds at Al Mina in North Syria », Archaeologia 87, p. 19-78.

LÉCuYer (N.) 2004, « Le territoire de Potamia aux époques médiévale et moderne : acquis récents », CCEC 34, p. 11-29.
LÉCUYER (N.) 2006, « Marqueurs identitaires médiévaux et modernes sur le territoire de Potamia-Agios Sozomenos ", dans S. Fourrier, G. Grivaud (éd.), Identités croisées en un milieu méditerranéen : le cas de Chypre (Antiquité-Moyen Âge), Mont-Saint-Aignan, p. 241-256.

Loomis (R.S.H.), 1959, « Arthurian Influence on Sport and Spectacle », dans R.S. Loomis (éd.), Arthurian Literature in the Middle Ages: A Collaborative History, Oxford, p. 553-559.

Ludolphus de Sudheim, 1884, De itinere Terre Sancte, éd. G.A. Neumann, Archives de l'Orient Latin 2, p. 305-377.

Malamut (É.), 1988, Les îles de l'Empire byzantin, VIII $^{e}$-XII ${ }^{e}$ siècles, Paris, 2 vol.

Malamut (É.), 2012, « Chypre nouvelle province byzantine », dans Durand, Giovannoni 2012, p. 96-102.

MAIER (F.), 1979, « Excavations at Kouklia (Palaepahos). Tenth Preliminary Report: Seasons 1977 and $1978 », R D A C$, p. 168-176.

Megaw (A.H.S.), 1951, « Three Medieval Pit Groups from Nicosia », RDAC 1937-1939, p. 145-168.

Megaw (A.H.S.), 1972, « Supplementary Excavations on a Castle Site at Paphos, Cyprus, 1970-1971 », DOP 26, p. 332-343.

Megaw (A.H.S.), Rosser (J.), 2001, « A Watchtower before Pafos Castle », RDAC, p. 319-331.

Mogabgab (T.), 1937-1939, « Excavations and Researches in Famagusta, 1937-1939 », RDAC, p. 181-190.

Nepoti (S.), 1991, Ceramiche graffite della donazione Donini Baer, Faenza.

Ostrogorsky (G.), 1983, Histoire de l'État byzantin, Paris.

Otten-Roux (C.), 1996, « Les relations économiques entre Chypre et le royaume arménien de Cilicie d'après les actes notariés (1270-1320) », dans N. Garsoïan (dir.), L'Arménie et Byzance. Histoire et culture, Paris, p. 157-179.

Papacostas (T.), 2006, « Architecture et communautés étrangères à Chypre $\mathrm{au} \mathrm{XI}^{\mathrm{e}}$ et 
au XII ${ }^{\mathrm{e}}$ siècles ", dans S. Fourrier, G. Grivaud (éd.), Identités croisées en un milieu méditerranéen : le cas de Chypre (Antiquité Moyen Âge), Mont-Saint-Aignan, p. 223-240.

Papademetriou (E.), 2005, Modern Glazed Pottery of Cyprus. Lapithos Ware, Nicosie.

PAPANIKola-BAKIRTZI (D.), 1989, « Medieval Pottery from Enkomi, Famagusta », dans V. Déroche, J.-M. Spieser (éd.), Recherches sur la céramique byzantine, BCH Suppl. XVIII, p. 233-246.

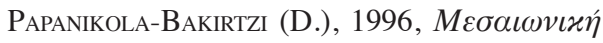

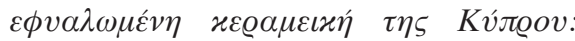

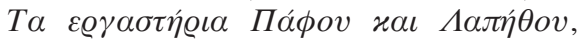
Thessalonique.

PAPANikola-BaKirtzi (D.), 1997, « E $\phi v \alpha \lambda \omega-$

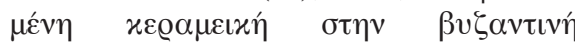

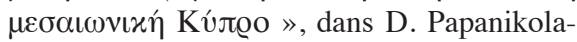
Bakirtzis, M. Iakovou (éd.), $B v \zeta \alpha v \tau \iota v \eta ́$

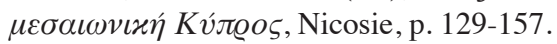

Papanikola-BakiRtzi (D.), 2004, Colours of Medieval Cyprus, Nicosie.

Patitucci-Uggeri (S.), 1985, «La protomaiolica del Mediterraneo orientale in rapporto ai centri di produzione italiani », $C C A B 32$, p. 337-402.

Piltz (E.), 1996, The Von Post Collection of Cypriote Late Byzantine Glazed Pottery, Jonsered.

Pringle (D.), 1982, « Some More Protomaiolica from “Atlit (Pilgrim's Castle) and a Discussion of its Distribution in the Levant », Levant 14, p. 104-117.

Pringle (D.), 1985, « Medieval Pottery from Caesarea: the Crusader Period », Levant 17, p. 171-202.

Pringle (D.), 1986, « Pottery as Evidence for Trade in the Crusader States ", dans G. Airaldi, B.Z. Kedar (éd.), I Comuni italiani nel regno crociato di Gerusalemme, Gênes, p. 451-475.

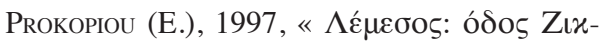

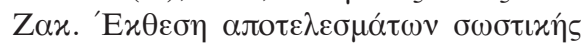

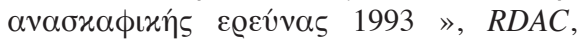
p. 285-322.
Raynaud de Lage (G.), 1976, « Les romans antiques et la représentation de l'Antiquité », dans G. Raynaud de Lage, Les premiers romans français, Genève, p. 127-160.

RedFord (S.), 2004, « On Sāqīs and Ceramics: Systems of Representation in the Northeast Mediterranean ", dans D.H. Weiss, L. Mahoney (éd.), France and the Holy Land. Frankish Culture at the End of the Crusades, Baltimore et Londres, p. 282-312.

RÉGNIER-Bohler (D.) éd., 1995, Splendeurs de la cour de Bourgogne, Paris.

Riavez (P.), 2001, "'Atlit - ceramica Port St. Symeon 1217-1291, graffite 'crociate' del Mediterraneo orientale », Archeologia Medievale 28, p. 505-532.

RichaRd (J.), 1979, « Le peuplement latin et syrien en Chypre au XIII ${ }^{\mathrm{e}}$ siècle », ByzForsch 7 , p. 157-173 [repr. dans J. Richard, Croisés, missionnaires et voyageurs. Les perspectives orientales du monde latin médiéval, Londres, 1983, étude ${ }^{\circ}$ VII].

Rudt de Collenberg (W.H.), 1982, « Le déclin de la société franque de Chypre entre 1350 et

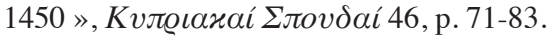

SCAnglon (G.T.), 1967, « Fustat Expedition: Preliminary Report, 1965, Part II », JARCE 6, p. 65-86.

Scanglon (G.T.), 1971, « The Fustat Mounds, A Shard Count 1968 », Archaeology 24/3, p. 220-233.

SCANGlon (G.T.), 1984, « Mamluk Pottery: More Evidence from Fustat », Muqarnas 2, p. 115-126.

Siviero (G.B.), 1986, « La ceramica graffita veneta », Atti del' XIX Convegno Internazionale della Ceramica, Albisola, p. 29-41.

Stanesco (M.), 1998, Lire le Moyen Âge, Paris.

STERn (E.), 2008, « Production and Export of $13^{\text {th }}$-century CE Cypriot Pottery: the Evidence from the Crusader Kingdom of Jerusalem », RDAC, p. 455-469.

Stern (E.), Waksman (S.Y.), 2003, « Pottery from Crusader Acre: a Typological and 
Analytical Study », dans Bakirtzis 2003, p. 167-180.

VAllauri (L.), 2004, « Céramiques en usage à Potamia-Agios Sozomenos de l'époque médiévale à l'époque ottomane », CCEC 34, p. 223-235.

Vallauri (L.), Démians d’Archimbaud (G.), 2003, « La circulation des céramiques byzantines, chypriotes et du Levant chrétien en Provence, Languedoc et Corse du $\mathrm{X}^{\mathrm{e}}$ au XIV ${ }^{\mathrm{e}}$ siècle », dans Bakirtzis 2003, p. 137-152.

Violaris (Y.), 2004, « Excavations at the Site of Palaion Demarcheion, Lefkosia », CCEC 34, p. 69-80.

Waksman (S.Y.), 2002, « Céramiques levantines de l'époque des croisades : le cas des productions de Beyrouth ", Revue d'Archéométrie 26, p. 67-77.

Waksman (S.Y.), François (V.), 2004-2005, «Vers une redéfinition typologique et analytique des céramiques byzantines du type Zeuxippus Ware », BCH 128-129, p. 629-724.

Waksman (S.Y.), Teslenko (I.), Zelenko (S.M.), 2009, « Glazed wares as main cargoes and personal belongings in the Novy Svet shipwreck (13th c. AD, Crimea): a diversity of origins investigated by chemical analysis », dans J. Zozaya, M. Retuerce, M.A. Hervás, A. de Juan (éd.), Actas del VIII Congreso Internacional de Cerámica Medieval en el Mediterráneo, Ciudad Real-Almagro del 27 de febrero al 3 de marzo de 2006, Ciudad Real, vol. II, p. 851-856.

Waksman (S.Y.), 2011, « Ceramics of the "Serçe Limanı Type' and Fatimid Pottery Production in Beirut », Levant 43/2, p. 201-212.

WARTburg (M.-L. von), 1997a, « Medieval Glazed Pottery from the Sanctuary of Aphrodite at Palaipaphos (Site TA). A Preliminary Survey », RDAC, p. 184-193.

WARTBurg (M.-L. von), 1997b, « Lemba Ware Reconsidered », RDAC, p. 323-340.
WARtburg (M.-L. von), 1998, « Mittelalterliche Keramik aus dem Aphroditeheiligtum in Palaipaphos (Grabungsplatz TA) », Archäologischer Anzeiger, p. 133-165.

WARTBURG (M.-L. von), 2001, «"Hochzeitspaare" und Weintrinker Zu Bildmotiven der mittelalterlichen Keramik Cyperns », Zona Archaeologia, Festschrift für Hans Peter Isler zum 60. Geburtstag, Bonn, p. 457-465.

Wartburg (M.-L. von), 2003, « Cypriot Contacts with East and West as reflected in Medieval Glazed Pottery from the Paphos Region », dans Bakirtzis 2003, p. 153-166.

Wartburg (M.-L. von), Portmann (A.), Wild (F.), Stockhause (S.), Berke (H.), 2010, "'Islamic' Table Ware found in Cyprus: Study and Analysis of Fabric and Glazes », RDAC, p. 389-410.

Wartburg (M.-L. von), Violaris (Y.), 2009, " Pottery of a 12th Century Pit from the Palaion Demarcheion site in Nicosia: a Typological and Analytical Approach to a closed assemblage», dans J. Zozaya, M. Retuerce, M.A. Hervás, A. de Juan (éd.), Actas del VIII Congreso Internacional de Cerámica Medieval en el Mediterráneo, Ciudad Real-Almagro del 27 de febrero al 3 de marzo de 2006, Ciudad Real, vol. I, p. 249-264.

WeYl CARr (A.), 2005, " Art », dans A. Nicolaou-Konnari, Ch. Schabel (éd.), Cyprus, Society and Culture 1191-1374, Leyde et Boston, p. 285-328.

Woledge (B.), 1953, « La légende de Troie et les débuts de la prose française », Mélanges M. Roques, Bade et Paris, vol. II, p. 313-324.

YотA (É.), 2012, « Manuscrits grecs chypriotes : culture écrite, images et marques de possesseurs ", dans Durand, Giovannoni 2012, p. 138-142. 

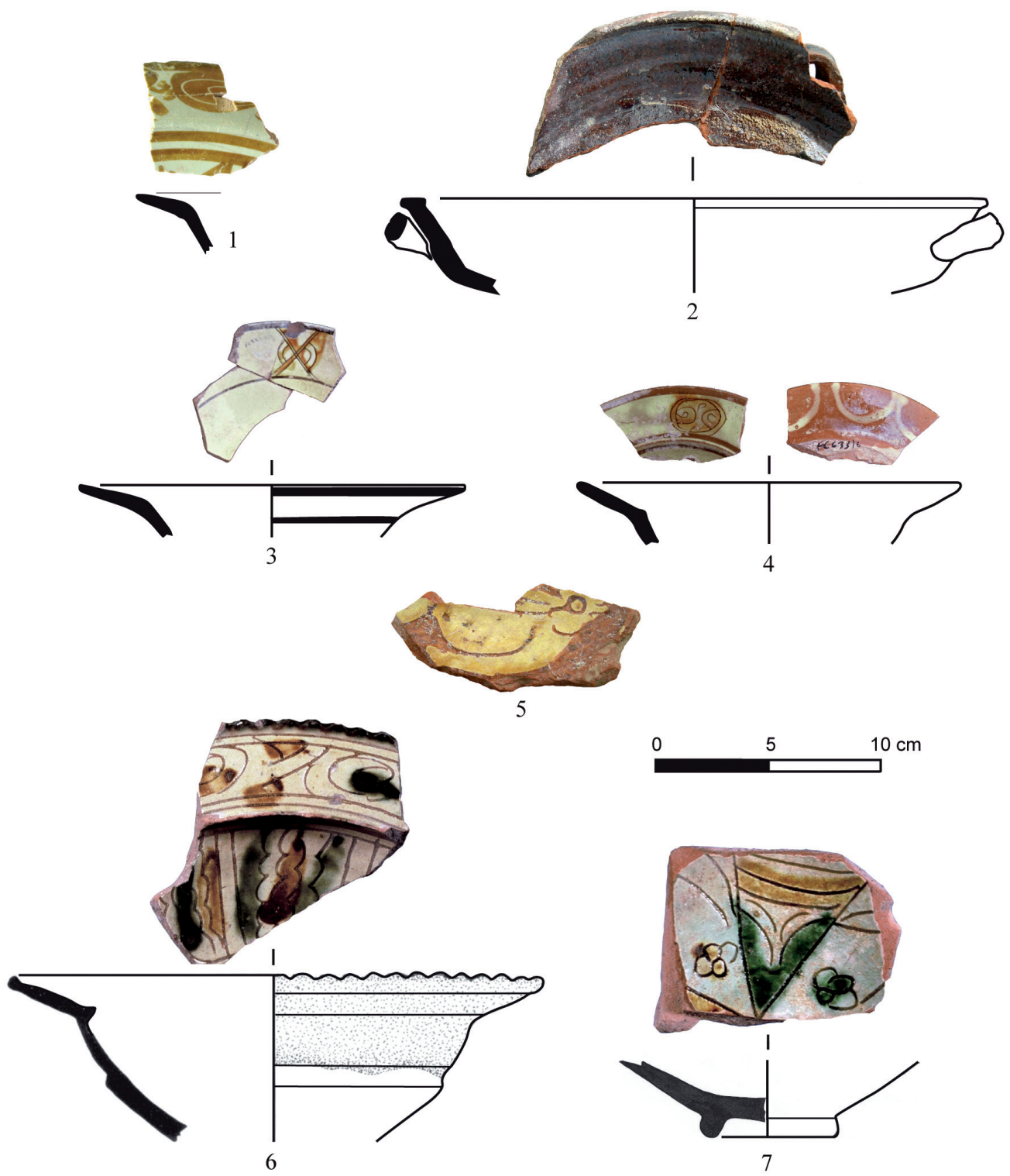

Figure 1.

1. Coupe peinte au lustre métallique de Syrie ou d'Égypte, XII ${ }^{\mathrm{e}}$ s. (Paphos, Fabrika);

2. Jatte culinaire glaçurée de Beyrouth, $\mathrm{XII}^{\mathrm{e}} \mathrm{s}$. (Paphos, Fabrika);

3, 4. Zeuxippus Wares, fin XII -troisième quart du XIII ${ }^{\mathrm{e}} \mathrm{s}$. (Paphos);

5. Aegean Ware, fin XII -troisième quart du XIII ${ }^{\mathrm{e}}$ s. (Paphos, Fabrika);

6,7 . Céramiques «d'Al-Mina », fin XIII ${ }^{\mathrm{e}} \mathrm{s}$. 


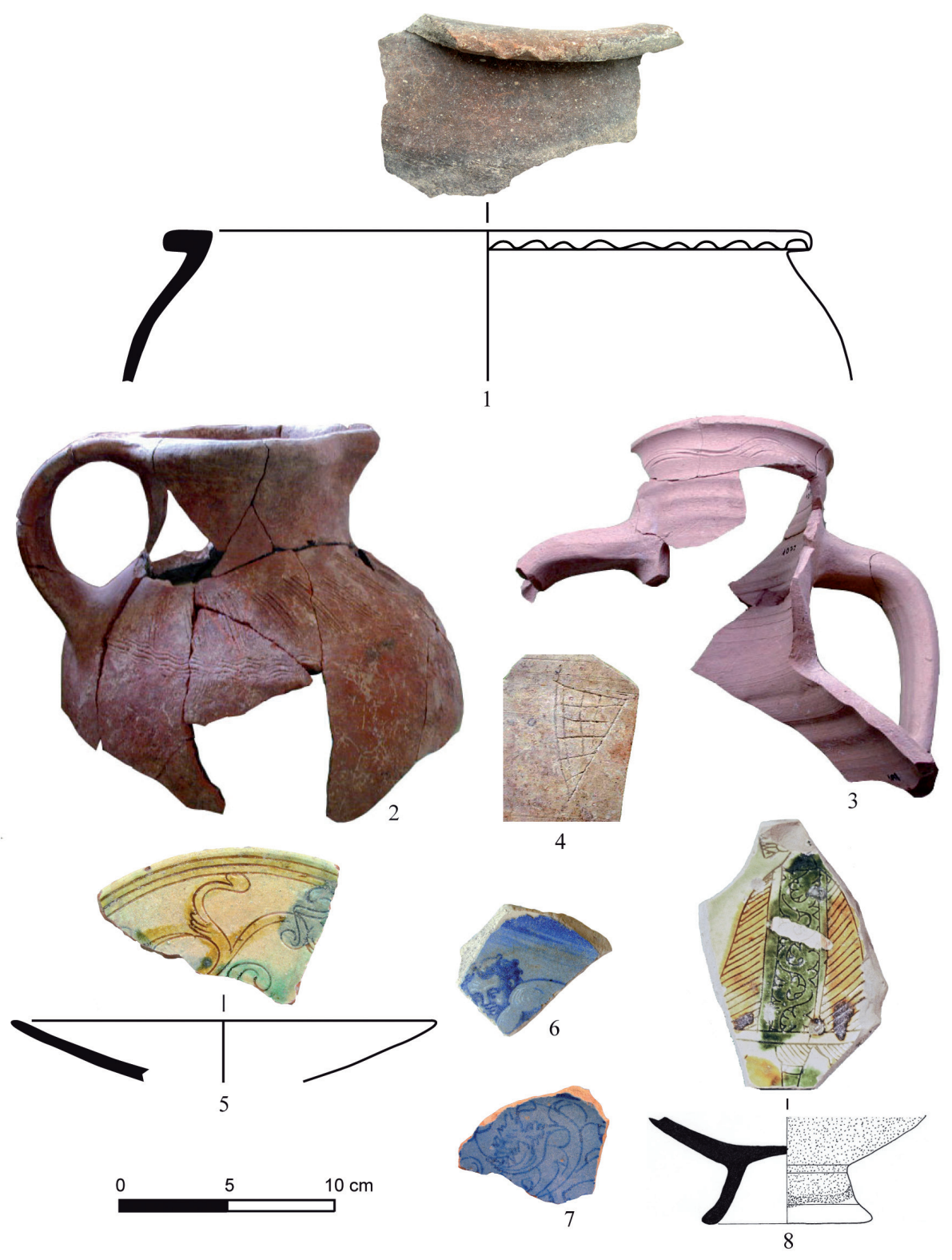

Figure 2.

1. Marmite modelée, XIV ${ }^{\mathrm{e}} \mathrm{s}$. (Paphos, Fabrika);

2. Cruche modelée, XIV s. (Potamia);

3 , 4. Jarre à pâte claire et blason incisé, xIv ${ }^{\mathrm{e}} \mathrm{s}$. (Potamia);

5. Graffita tarda d'Italie du Nord, XVI s. (Paphos, Fabrika);

6, 7. Faïences de Ligurie ou de Padoue (Potamia et Fabrika);

8. Céramique de Lapithos, $X v^{\mathrm{e}} \mathrm{s}$. 